\title{
In situ Composite Ion-Triggered Gellan Gum Gel Incorporating Amino Methacrylate Copolymer Microparticles: A Therapeutic Modality for Buccal Applicability
}

\section{Enas Elmowafy, Marco Cespi, Giulia Bonacucina \& Mahmoud E. Soliman}

To cite this article: Enas Elmowafy, Marco Cespi, Giulia Bonacucina \& Mahmoud E. Soliman (2019): In situ Composite Ion-Triggered Gellan Gum Gel Incorporating Amino Methacrylate Copolymer Microparticles: A Therapeutic Modality for Buccal Applicability, Pharmaceutical Development and Technology, DOI: 10.1080/10837450.2019.1659314

To link to this article: https://doi.org/10.1080/10837450.2019.1659314

Accepted author version posted online: 22

Aug 2019.

Submit your article to this journal

Q View related articles $\asymp$

View Crossmark data $₫$ 


\title{
In situ Composite Ion-Triggered Gellan Gum Gel Incorporating Amino Methacrylate
} Copolymer Microparticles: A Therapeutic Modality for Buccal Applicability

\author{
Enas Elmowafy ${ }^{a}$, Marco Cespi $^{b}$, Giulia Bonacucina $^{b}$, and Mahmoud E. Soliman ${ }^{a}$
}

${ }^{a}$ Department of Pharmaceutics and Industrial Pharmacy, Faculty of Pharmacy, Ain

Shams University, Cairo, Egypt,Monazzamet Elwehda Elafrikeya Street, Abbaseyya, Cairo, Egypt, P.O. 11566

${ }^{b}$ School of Pharmacy, University of Camerino, Camerino, Italy Via S. Agsotino 1, 62032

\section{* Corresponding author:}

Enas Elmowafy

Department of Pharmaceutics and Industrial Pharmacy,

Faculty of Pharmacy, Ain Shams University, Cairo, 11566, Egypt

Tel.: +2 01005164052 Fax: +20 20224011507 .

Email: enasmostafa@pharma.asu.edu.eg 


\begin{abstract}
The aim of the current investigation is to delineate the buccal applicability of an in situ composite gel containing aceclofenac (AC) amino methacrylate copolymer microparticles (MPs), surmounting limitations of oral existing conventional therapy. AC Eudragit RL100 MPs were fabricated and statistically optimized using $2^{2} 4^{1}$ factorial design. Better buccal applicability and enhanced localization were achieved by combining the optimum MPs with in situ ion-activated gellan gum gel. The crosslinking and gelation of in situ gel were investigated by morphological and solid state characterizations. Suitability for buccal delivery and in vivo therapeutic efficacy in inflammation model of rats were also assessed. Results showed that the best performing formula displayed particle size (PS) of $51.00 \mu \mathrm{m}$ and high entrapment efficiency (EE\%) of 94.73\%. MPs were successfully entrapped inside the gel network of the composite system. Gelation tendency, $\mathrm{pH}$, shear-thinning properties and mucoadhesivity of the prepared in situ composite gel guaranteed its buccal suitability. Sustained AC release features and promising in vitro anti-arthritic response were also demonstrated. Moreover, consistent and prolonged in vivo anti-inflammatory effect was achieved, relative to standard AC. Taken together; this study proves the potential of in situ composite gel as an appropriate therapeutic proposal for AC buccal delivery.
\end{abstract}

\title{
Keywords
}

Eudragit RL100, Microparticles, Gellan gum, Ion-triggered gel, Aceclofenac, Buccal delivery 


\section{Introduction}

Versatile polymers-based systems have remarkable aptitude for drug delivery via various mucosal surfaces with reproducible qualities, offering meaningful therapeutic modulation prospects (Momoh et al. 2014; Ferreira et al. 2015; Elmowafy E et al. 2017; Ferreira et al. 2018; Elmowafy E, Abdal-hay A, et al. 2019; Elmowafy EM et al. 2019). Among different utilized polymers in drug delivery systems (DDS) fabrication, poly(methacrylates) polymers (e.g. eudragit RL, eudragit RS) have been widely employed for the last decades for bioencapsulation and controlled drug release (Cui et al. 2009; Animesh et al. 2012; Soliman ME, Elmowafy, E.M., Almogerbi, A., Mansour, S.A., \& Shamy, A.A 2016).

Considering these peculiarities, tailored poly(methacrylates) polymers based DDS have been well-investigated in various routes including the buccal route (Reddy et al. 2011; Morales et al. 2013; Mouftah et al. 2016). In particular, cationic aminomethacrylate copolymers (e.g. eudragit RL100) could allow strong binding with negatively charged glycoproteins of mucus via non-covalent bonds and hence, longer residence time on the buccal mucosal surface (Morales et al. 2013; Garipova et al. 2018). Indeed, the buccal route offers great promises in drug delivery, owing to high vasculature, and high permeability of the buccal mucosa increased patient compliance and convenient application (Reddy et al. 2011; Khames 2019). Compared to the dermal route, the buccal mucosa is $4-4000$ times more permeable than the skin (Galey et al. 1976). Thus, accelerated drug absorption and augmented therapeutic effect could be realized; issues of meaningful significance for either systemic effect or localized effect in buccal cavity tissues for the treatment of mouth ulcers and periodontal disease (e.g. gingivitis) (Hassan M et al. 2011; Sanghai et al. 2016). 
Depending on their outstanding merits, microparticles (MPs) have been widely employed as buccal carriers, due to their capability of providing intimate contact with the large surface area of buccal mucosa, sustained drug release and better patient acceptability (Patel VF et al. 2011). Furthermore, MPs could avoid local irritation and uncomfortable mouthfeel of a foreign body within the oral cavity (Sudhakar et al. 2006; Reddy et al. 2011). However, several issues should be considered during their buccal administration, i.e. ease of applicability and prolonged buccal retention. To fulfill such purposes, various delivery modes of MPs have been investigated, including 'tablets, aqueous suspensions, gels and aerosols (Patel VF et al. 2011; Reddy et al. 2011).

Undoubtedly, implementing gel systems; conventional preformed or in situ gels as MPs carriers could have added merits over other alternatives. From delivery perspective, the first one; conventional preformed gels have the following attributes: (i) providing thick gel barrier over buccal mucosa with prolonged retention in the oral cavity and (ii) avoidance of quick and premature removal by salivation and other mechanical activities like tongue movement and swallowing (Cid et al. 2012; Suresh and Manhar 2014). Unfortunately, the application of an accurate dose of these preformed gels might be inconvenient, because of the possible delivery of variable drug amounts (Russo et al. 2016). In addition, frequent dosing is required to ensure the intimate contact between the preformed gel and the absorbing site of application.

On the other hand, in situ gels are being adopted as a lucrative option by exhibiting a solto-gel phase transition, combining the advantages of solutions and gels in the same formulation. Thus, topical instillation of in situ gels in the form of sols could provide the following benefits: (i) accurate dose application, (ii) enhanced spreadability, (iii) optimal coating of the absorbing 
tissue, and (iv) prolonged residence time (Harish et al. 2009; Ibrahim et al. 2012; Irimia et al. 2018) .

In the current investigation, ion-triggered gellan gum gel has been chosen among other in situ gels to facilitate the intimate and prolonged buccal application of mucoadhesive aminomethacrylate copolymer, eudragit RL100, based MPs, owing to the following features: (i). mucoadhesiveness of the anionic gellan gum (Elmowafy E et al. 2014), (ii) biodegradability, and (iii) capability of formation of ionotropically cross-linked gels in the presence of multivalent cations (Jana, Das, et al. 2013; Elmowafy E et al. 2014; Prezotti et al. 2014; Boni et al. 2016).

The in situ ion-triggered gel is designed by incorporating calcium chloride (chemical source of calcium ions and cross-linker) and sodium citrate (complexing agent for free calcium) into the gellan gum base. Sodium citrate sequesters free calcium ions, forming complexed calcium form that is then liberated via complex dissociation in the physiological conditions in the buccal cavity, in order to promote instantaneous gelation of gellan gum (Miyazaki et al. 1999; Nagarwal and Pandit 2008). From a prolonged delivery perspective, blending gellan gum and HPMC; a semisynthetic cellulose polymer is well-demonstrated in literature in order to conceivably enhance gel structure and strength, augment viscosity and mucoadhesion and retard drug release (Harish et al. 2009; Xie et al. 2019).

Therefore, in this work, the suitability of the aforementioned in situ composite system for buccal delivery of a non-steroidal anti-inflammatory drug; aceclofenac (AC) has been exploited, surmounting shortcomings of its oral formulae, viz. short elimination half-life (4h) and considerable gastrointestinal disturbances (nausea, diarrhea and peptic ulceration) (Srinivas et al. 2010; Fathalla et al. 2014). Despite many previous works refer that AC topical application presents a potential treatment of the inflammatory conditions reducing systemic side-effects 
(Dasgupta et al. 2013; Raj et al. 2016; Ilić et al. 2018), sustained release in situ composite systems intended for AC buccal delivery is still not available. To the best of our knowledge, this study elucidates for the first time the critical parameters affecting in vitro physicochemical attributes, sustained release and in vivo performance of the in situ composite systems; crosslinking, gelation, and possible interaction between cationic eudragit RL100 and anionic gellan gum in the composite system via morphological and solid-state characterization. The mucoadhesiveness, in vitro anti-arthritic activity and in vivo anti-inflammatory activities of the proposed system were also evaluated to give an indication about the efficiency of in situ iontriggered gel as a buccal drug delivery system.

\section{Materials and Methods}

\section{Materials}

AC (99.9\% purity) was gifted from Bristol-Myers Squibb Company, Cairo, Egypt. Gellan gum, polyvinyl alcohol (PVA), Tween 80 (T80), Poloxamer 188 (P188), Myrj 59 (M59) and carrageenan were purchased from Sigma Chemical Co., St. Louis, USA. Eudragit RL100 was supplied by Evonik Pharma, GmbH, Darmstadt, Germany. Dichloromethane (DCM), potassium dihydrogen phosphate, disodium hydrogen phosphate, calcium chloride, sodium citrate, and sodium chloride were supplied by ADWIC, El-Nasr chemical Co. Cairo, Egypt. HPMC (Methocel E5, low viscosity) was kindly supplied by E.I.P.I.C.O. Company, Cairo, Egypt. Spectra/Por dialysis membrane, 12.000-14.000 molecular weight cut off was purchased from Spectrum Laboratories Inc., Rancho Dominguez, Canada.

\section{Preparation and Statistical Optimization of Aceclofenac Eudragit RL100 Microparticles}

AC Eudragit RL100 MPs were prepared using an emulsion solvent evaporation technique as previously reported (Harsh et al. 2014). In brief, fixed amounts of Eudragit RL100 and AC, 
dissolved in dichloromethane, were dropped in an aqueous solution containing O/W stabilizer. The effect of different formulation variables on EE \% and PS of MPs was optimized using $4^{1} 2^{2}$ full factorial design. Three factors were estimated: two factors with two levels (A: Drug amount) and (B: Surfactant concentration) while the third with four levels (C: type of surfactant) as displayed in Table 1. The quantity of Eudragit RL100 (500 mg) and organic to aqueous volume ratio $(1: 10)$ were kept constant in all experimental formulae.

[Table 1 near here]

Determination of Entrapment Efficiency \% and Particle Size of Aceclofenac Eudragit RL100

\section{Microparticles}

An accurately weighed amount of AC Eudragit RL100 MPs was dissolved in methanol by vortexing and assayed spectrophotometrically (UV-1601 PC, Shimadzu, Kyoto, Japan) at 274 $\mathrm{nm}$ after appropriate dilution. Entrapment efficiency was determined by dividing the actual entrapped amount of AC to the total amount of AC. PS of MPs aqueous dispersions using deionized water was measured by a laser diffraction particle size analyzer (Mastersizer X, Malvern Instruments Ltd., UK) (Madhusudhan et al. 2010). The observed average particle size was expressed as volume mean diameter $(\mathrm{VMD})$ in $\mu \mathrm{m}(\mathrm{D}[4,3])$. SPAN as an indicator of particle size distribution was also measured.

\section{Preparation of Plain In Situ Gel}

Preliminary trials were conducted for screening various concentrations of gellan gum $(0.1$ $0.5 \%)$, sodium citrate $(0.1-0.2 \%)$ and calcium chloride $(0.05-0.1 \%)$. Based on these trials, the in situ gel in liquid form was prepared by placing equal concentrations of gellan gum and HPMC $(0.5 \%)$ in a well stirred and heated $0.2 \% \mathrm{w} / \mathrm{v}$ sodium citrate solution (at $\left.90^{\circ} \mathrm{C}\right)$. After cooling to below $40^{\circ} \mathrm{C}, 0.1 \%$ of calcium chloride was added into the sol (Harish et al. 2009). Freeze-drying 
of the in situ gel was performed (freeze drying under vacuum for $48 \mathrm{~h}$ using Chirst, alpha 1-2LD plus, Germany), in order to conduct in vitro characterization tests, namely, morphologic analysis, DSC and FT-IR (Kesavan et al. 2010; Xie et al. 2019) .

\section{The Gelling Capacity, Gelation Time and pH of Plain In Situ Gel}

The gelling capacity of in situ gel was determined visually using tube inversion method by placing a glass vial containing $2 \mathrm{ml}$ of freshly prepared simulated salivary fluid at $\mathrm{pH} 6.8$ and the produced gellan gum sol in a water bath and adjusting its temperature at $37 \pm 0.5{ }^{\circ} \mathrm{C}$. The gel formation (speed "gelation time" and extent "gel stiffness") and time interval for which formulation remains as gel were assessed according to the following gelation capacity scoring grades (Kesavan et al. 2010; Swain et al. 2019):

(+) Rapidly eroded in situ gel that forms after a few minutes

(++) Immediately formed in situ gel that remains for few hours

$(+++)$ Immediately formed in situ gel that remains for an extended period of time pH was determined using a pH meter (model 3510, Jenway, Burlington, NJ).

\section{Preparation of In Situ Composite Gel (In Situ Gel Incorporating AC Eudragit RL100 MPs)}

The optimized AC Eudragit RL100 MPs were incorporated into in situ gellan gum gel as follows: precise weight of AC MPs was dispersed in the produced sol using a magnetic stirrer and thoroughly mixed till even distribution of MPs throughout in situ gel can be visually observed. The impact of MPs incorporation on $\mathrm{pH}$ and gelling capacity and time was demonstrated, via measuring $\mathrm{pH}$ value and visual assessment of the formed in situ composite gel respectively. 


\section{Viscosity Determination}

The viscosity values of plain gel and in situ composite gel were measured using a DV-E Viscometer (Brookfield Engineering Laboratories, Middleboro, MA, USA) using a 52 spindle at speeds ranging from 0.05 to $100 \mathrm{rpm}$ at $37^{\circ} \mathrm{C}$ over $10 \%$ torque. By plotting viscosity values (Pa.s) versus angular velocity $(\mathrm{rad} / \mathrm{sec})$, the flow pattern was checked.

\section{Mucoadhesivity Assessment}

Based on the viscosity measurements, the mucoadhesive potential of the plain gel and in situ composite gel was evaluated. The change in the viscosity of the mucin-gel dispersion from that of each component alone was recorded (Hassan EE and Gallo 1990; Fini et al. 2011; Soliman ME et al. 2018). The gel was allowed to be in contact with mucin (15\% w/v) for an appropriate contact time ( 3 min). The difference between $\eta_{\text {mixture }}$ and the sum of $\left.\left(\eta_{\text {gel }}+\eta_{\text {mucin }}\right)\right]$ is equal to the contribution of the viscosity of the mucin-gel association $(\Delta \mathrm{\eta}$; component of mucoadhesion) using Eq. (1) (Fini et al. 2011):

$\Delta \eta=\left[\eta_{\text {mixture }}-\left(\eta_{\text {mucin }}+\eta_{\text {gel }}\right)\right]$

where: $\eta_{\text {mucin }}, \eta_{\text {gel }}$, and $\eta_{\text {mixture }}$ are the viscosity coefficients of mucin, gel and mucin/gel mixture respectively. The results were expressed in terms of the ratio between the component of mucoadhesion and viscosity of gellan gum gel $\left(\Delta \eta / \eta_{\text {gel }}\right)$. Each recorded viscosity value was the mean of three consecutive determinations.

\section{Characterization of AC Eudragit RL100 MPs and In Situ Composite Gel}

\section{Morphologic Analysis of the Microstructure of MPs and In Situ Composite Gel}

Morphological examination of MPs and freeze dried in situ composite gel (composite gel powder) was conducted by means of scanning electron microscopy (SEM) (Zeiss, Germany). A small amount of powder was spread on an aluminum stub and, after gold sputtering, was 
visualized at $20 \mathrm{kV}$ acceleration voltages under argon atmosphere and images were observed under various magnifications.

\section{In vitro Drug Release and Release Kinetics}

In vitro drug release behavior, similar to the one attempted by (Elkomy et al. 2017), was done. In details, an accurate volume of in situ composite gel equivalent to $2 \mathrm{mg} \mathrm{AC}$ was loaded in cylinders of cellulose acetate membrane and placed in $100 \mathrm{ml}$ simulated salivary fluid (pH 6.8) maintained at $37{ }^{\circ} \mathrm{C}$ in shaking water bath at $50 \mathrm{rpm}(\mathrm{Chan}$ et al. 2019). The release of AC powder, AC loaded in situ gel (control gel) and AC Eudragit RL100 MPs were also performed for comparison. Control gel was prepared by dissolving AC in the minimum amount of ethanol followed by gradual mixing to the preformed stirred gel. At preplanned time intervals, $1 \mathrm{~mL}$ was sampled and replaced with an equal volume of fresh media. The quantity of AC released was determined using UV spectrophotometry (UV-1601 PC, Shimadzu, Kyoto, Japan) at 274 nm. In vitro release profiles of AC were fitted to different kinetic models (zero order, first order, and Higuchi's model), and the release mechanism was analyzed using the Korsmeyer-Peppas model. Solid State Characterization

Differential Scanning Calorimetry (DSC):The thermal properties of AC, Eudragit RL100, 1:1 AC:Eudragit RL100 physical mixture, selected AC Eudragit RL100 MPs (PV3), gellan gum and freeze dried in situ composite gel were estimated using DSC (Shimadzu-DSC 60, Kyoto, Japan) at a heating rate of $10^{\circ} \mathrm{C} /$ min over a temperature range of $25^{\circ} \mathrm{C}-300^{\circ} \mathrm{C}$.

Fourier Transform Infrared (FT-IR) Spectroscopy: FT-IR spectra of AC, Eudragit RL100, 1:1 AC: Eudragit RL100 physical mixture, selected AC Eudragit RL100 MPs (PV3), gellan gum and freeze dried in situ composite gel were assessed by FT-IR spectrophotometer using $\mathrm{KBr}$ disc method (Nicolet 6700 FT-IR; Thermal Scientific; Class 1 laser product; USA). 


\section{In vitro Anti-arthritic Activity}

The anti-denaturation assay using bovine serum albumin was carried out on selected AC Eudragit RL100 MPs (PV3), plain gellan gum gel and in situ composite gel as previously described (Elisha et al. 2016). Diclofenac sodium was used as a positive control (final concentrations of $7.81-1000 \mu \mathrm{g} / \mathrm{mL})$. The percentage inhibition of protein denaturation was computed for all studied concentrations by using Eq. (2):

$\%$ inhibition $=\frac{A b s_{\text {control }}-A b s_{\text {test }}}{A b s_{\text {control }}} \times 100$

where: $\mathrm{Abs}_{\text {control }}$ is the absorbance of albumin solution at $\lambda=660 \mathrm{~nm}$ for $100 \%$ denatured protein and $\mathrm{Abs}_{\text {test }}$ is the absorbance of albumin mixed with tested samples.

The IC50 was calculated from a graph depicting protein inhibition against the different concentrations. The values of concentrations $(7.81-1000 \mu \mathrm{g} / \mathrm{mL})$ were for the anti-inflammatory drugs (diclofenac and aceclofenac). Plain gel was tested using the same concentration of in situ composite gel without adding AC loaded MPs (PV3).

\section{In vivo Anti-inflammatory Activity}

Assessment of the anti-inflammatory activity of in situ composite gel was performed using the carrageenan-induced rat-paw edema model as described previously (Patel B et al. 2011; Jana et al. 2014). Male Sprague Dawley rats (weights, 150-200 g) were used in the study in accordance with a protocol approved by the Ethical Committee of Faculty of Pharmacy, Ain-Shams University on the use of animals. The fasted animals were divided into 4 groups $(n=6)$ and treated as follows:

Group 1 (control group): received oral administration of $2.5 \mathrm{ml}$ of $0.5 \%$ sodium carboxymethyl cellulose (Na CMC) aqueous solution. 
Group 2 (control group): received a buccal application of plain gellan gum gel (in situ gel in liquid form).

Group 3 (standard group): received oral administration of pure aceclofenac $(10 \mathrm{mg} / \mathrm{kg}$ body weight) with $0.5 \% \mathrm{Na} \mathrm{CMC}$ aqueous solution.

Group 4 (test group): received a buccal application of in situ composite gel (equivalent to AC 10 $\mathrm{mg} / \mathrm{kg}$ body weight) (in situ gel in liquid form).

Edema paw volumes were measured before and after carrageenan administration at different time intervals with a plethysmometer (UGO-Basile, 7140, Comerio, Italy). \% inhibition of paw edema was calculated for all time intervals by using Eq. (3):

$\%$ inhibition $=\left(1-\frac{D_{t}}{D_{c}}\right) \times 100$

Where: $D_{t}$ is the difference in paw volume in drug-treated groups and $D_{c}$ is the difference in paw volume in control groups.

\section{Statistical Analysis}

All measured data are expressed as mean \pm standard deviation (SD) of three or six independent experiments. The factorial design was analyzed using Design-Expert® v.8.0.7.1 (Stat-Ease, Inc., Minneapolis, MN, USA), For statistical significance, either Student's t-test or ANOVA was used to compare the mean values using Graph Pad Instat software program.

\section{Results and Discussion}

In the current study, an in situ composite gel, incorporating AC Eudragit R100 MPs was developed for optimal buccal applicability. As well-ascribed in literature, buccal administration of anti-inflammatory drugs is a desirable strategy for both local and systemic effects (Perioli et al. 2004; Perioli et al. 2007; Nazari et al. 2017). First, in preliminary work, many experiments were done to check the ideal concentration ranges of eudragit RL100 and different surfactants 
that fitted MPs formation and AC encapsulation (data not shown). The influence of inclusion of different stabilizers and their concentrations as well as different drug amounts on Eudragit RL100 MPs characteristics, viz. PS and EE\% was assessed using the statistical design for the conduct of experiments. Then, the best performing MPs were incorporated in the in situ gellan gel, originating composite system. Specifically, gellan gum based carriers, attempted in this work, have been considered lately for delivery of anti-inflammatory drugs through various routes, presenting promising therapeutic outcomes (Mahdi et al. 2014; Mahdi et al. 2016; Osmałek et al. 2017).

Preparation, Statistical Optimization, and Characterization of Aceclofenac Eudragit RL100

\section{Microparticles}

The emulsion solvent evaporation technique was selected for the fabrication of Eudragit RL100 MPs as it is a simple and commonly documented method for the preparation of MPs with high entrapment efficiency and satisfactory particle size for hydrophobic drugs. AC MPs were successfully fabricated using different stabilizers (PVA, P188, T80, and M59).

PS is a critical parameter to consider for MPs buccal delivery and should be optimized as it influences the mouthfeel and MPs palatability. Based on earlier literature, small particle size < $200 \mu \mathrm{m}$ would be more appropriate in terms of reduced grittiness and rough mouthfeel (Patil et al. 2016). In addition, the perception of oral grittiness was greatly reduced with increasing viscosity of MPs dispersion medium (Lopez et al. 2016). Therefore, the formulation of MPs in the gel system was attempted in our study to improve perceived grittiness besides ease of applicability as mentioned earlier. Besides, high values of EE\% are very crucial as it reflects the capacity of the prepared system to accommodate drug and the amount of MPs to be delivered. Thus, in this work, PS and EE \% were statistically optimized. 
Table 2 shows the composition and measured responses of the experimental runs. Statistical analysis of the $2^{2} 4^{1}$ factorial design (Table 3 ) exhibited that the fitted model was a three-factor interaction $(3 \mathrm{FI})$ model $(P<0.0001)$. High model accuracy values $(\mathrm{R} 2)$ of 0.9881 and 0.9970 were found with EE\% and PS respectively.

The $\%$ EE\% of AC varied greatly, ranging from $47.14 \%$ to $94.73 \%$ (Table 2). Fig. 1 illustrates the 3-D surface plots of EE\%. Based on the investigated design, the drug amount (A) was the most critical variable affecting $\mathrm{EE} \%(\mathrm{p}<0.0001)$. Increasing the drug amount was accompanied by increasing EE\%, indicating high drug-polymer affinity, augmenting its localization. As previously reported (Sharma et al. 2016), high drug-polymer affinity might oppose drug diffusion from the organic phase to the aqueous phase resulting in high encapsulation. In contrast, the EE\% and surfactant concentration (B) were negatively correlated. Being a hydrophobic molecule, AC might preferentially stay in the organic phase. However, the higher the surfactant concentration in the aqueous phase, the higher the diffusion of $\mathrm{AC}$ out from the organic phase, owing to its facilitated solubilization as micelles (Sharma et al. 2016).

The surfactant type (C) had also a significant impact on EE\%. The effect of various stabilizers on EE\% values of MPs could be ranked as follows: PVA > P188> M59> T80. The most efficient encapsulation was noticed with PVA and P188 that had shown similar trend on the effect of their concentration on $\mathrm{EE} \%$, ranging from $77.08 \%$ to $94.73 \%$ and from $73.42 \%$ to 92.34\% respectively (Table 2 , Fig. 1). This effect could be related to the alignment of their hydrophobic chains on the organic/aqueous interface, resulting in superior coverage of polymeric droplets and their steric stabilization. Thus, the drug may not partition out to the dispersion medium leading to higher EE\% values. 
As for M59 and T80, decreased values of EE\%, fitted between $56.42 \%$ and $89.60 \%$ and between $47.14 \%$ and $73.75 \%$ respectively, were revealed. This could be explained on the basis of the critical micelle concentration (CMC) values of both surfactants $(0.1 \%$ for M59(Goodhart and Martin 1962) versus $0.0014 \%$ for T80 (Wan and Lee 1974). Using concentrations higher than their CMC values could contribute to micelles formation in the aqueous phase and hence, poor AC encapsulation. The higher CMC value of M59 as compared to that of T80 might explain the higher EE\% values observed when using the former as a stabilizer.

Similarly, PS varied considerably $(51.00 \mu \mathrm{m}-135.39 \mu \mathrm{m})$ as shown in Table 2 and Fig. 2. It is worthy to note that PS values were $<200 \mu \mathrm{m}$, thus, possessing a masking effect on oral grittiness. Low span values ranged between $1.12 \pm 0.03$ and $4.83 \pm 0.54$, denoting narrow size distribution (data not shown). Drug amount (A) did not significantly influence PS ( $\mathrm{p}>0.05$ ), while both factors B and C were found to had significant effects on PS $(p<0.001)$. Regarding factor B, increasing concentration of either M59 or T80 yielded smaller particles and the reverse was true for both PVA and P188. Typically, the formation of small-sized particles upon increasing concentration of various surfactants could be explained by the alignment of surfactant molecules on the organic/aqueous interface. The resulted coverage of oil droplets and reduction of the interfacial tension between the organic and aqueous phase might have led to steric stabilization with a subsequent decrease in PS. However, for PVA and P188, their chosen concentrations might lead to deviation from the previously proposed effect and possible agglomeration. This could be justified based on possible gelatinization of PVA, owing to the possible formation of hydrogen bonds between intra- or inter- PVA molecules, and to the interparticle interaction of P188 chains (Blanco and Alonso 1997; Murakami et al. 1997). 
Interestingly, factor C "surfactant type" was found to be the most influential factor on PS $(\mathrm{p}<0.0001)$. In contrast with the other stabilizers, the PS variation was the least in case of MPs prepared with PVA, indicating its superior size-reducing effect. Furthermore, higher close PS values were obtained with MPs prepared using P188 and T80, whereas the highest PS values were obtained with MPs prepared using M59.

Due to such findings and considering suitable PS for buccal delivery and high EE\% (>90\%), MPs prepared using PVA (PV3 MPs) was selected for incorporation in the network of in situ gellan gum gel and further investigations (PV3 loaded gel).

[Table 2 near here]

[Table 3 near here]

[Figure 1 near here]

[Figure 2 near here]

Preparation of Plain and Composite In Situ Ion Triggered Gellan Gum Gel, Gelling Capacity, and $\mathrm{pH}$

The in situ ion-triggered gel comprising of a combination of gellan gum and HPMC and entrapping calcium ions was successfully prepared (El Maghraby et al. 2012). Herein, calcium ions were entrapped inside the gellan gum-HPMC sol as citrate complex. The complexing agent, sodium citrate reacted with calcium chloride, forming calcium citrate complex, ensuring sol fluidity. Then, calcium ions were liberated in the presence of simulated salivary fluid, due to $\mathrm{pH}$ change, resulting in ionic complexation and triggering instantaneous gelation (El Maghraby et al. 2012).

For in situ gelling systems, the gelling capacity is considered one of the prime perquisites. The in situ gel is proposed to be in the sol state at room temperature before 
administration, for ease of installation, and then undergoes rapid immediate gelation at the application site at $\mathrm{pH}$ 6.8. Additionally, the formed in situ gel should resist dissolution and maintain its gel consistency for an extended period of time. To achieve this target, optimum concentrations of the gel base, as well as complexing agents should be obtained.

In the preliminary experiments, it was observed that gellan concentrations below $0.25 \%$ were too low to form the gel network while those below $0.5 \%$ underwent gelation after few minutes ( $>5 \mathrm{~min})$ " + in gelling capacity scoring" or immediate gelation that remained for few hours "++". Thus, the optimum concentration of gellan gum was found to be $0.5 \%$, forming a stiff persistent gel within 15-20 seconds “+++”. Using a 1:1 ratio of gellan gum: HPMC yielded immediately formed resistant gel structure upon contact with simulated salivary fluid $\mathrm{pH} 6.8$ that was retained for an extended period of time. Likewise, the concentrations of complexing agents must ensure lack of free calcium ions in the vehicle, to remain in the fluid form prior to its application, but following sol instillation, the complex must be dissociated to release sufficient calcium ions to favor gelation. $0.1 \%$ calcium chloride and $0.2 \%$ sodium citrate concentrations were found to be satisfactory to achieve such purpose. Fig. 3 (a) depicts that the prepared in situ plain gel was homogenous and transparent showing no lumps. The gel homogeneity and absence of lumps were also confirmed for the composite system (selected PV3 loaded gel), as displayed in Fig. 3 (b), indicating that incorporating MPs inside in situ gel did not affect gelling capacity. Considering the satisfactory range of buccal $\mathrm{pH}(5.5-7.5)$, the prepared in situ plain gel was considered non-irritating and well tolerated with a $\mathrm{pH}$ value of $7.38 \pm 0.05$ (Patel et al. 2006; Dhiman et al. 2008). It is worth mentioning that the formation of a composite system by combining MPs with the in situ gel did not affect the $\mathrm{pH}$, showing similar value to that of plain in situ gel $(7.4 \pm 0.1 ; \mathrm{p}>0.05)$. 
[Figure 3 near here]

\section{Viscosity Determination}

Fig. 4 highlights the viscosity values obtained for plain and in situ composite gel (PV3 loaded gel). The results imply that pseudoplastic behavior was demonstrated as previously confirmed for gellan gum gels (Harish et al. 2009; Zhu et al. 2015). The shear-thinning behavior allowed the ease of spreading on buccal mucosa. The high viscosity of the gel also has been contributed to its mucoadhesive potential (Graciano et al. 2015). It is worthy to note that the obtained viscosity values were similar for each angular velocity tested $(p>0.05)$, indicating that incorporation of MPs in the in situ composite gel did not have an impact on its viscosity.

[Figure 4 near here]

\section{Mucoadhesivity Assessment}

Determination of mucoadhesive potential of formulations intended for mucosal delivery is very essential to evaluate their in vivo performance, intensifying their contact with the absorption surface (Elmowafy E and Soliman 2019). In particular, the attractive interaction between DDS and the buccal membrane is highly warranted for better mucosal penetration (Khutoryanskiy 2011) Utilizing the rheological characteristics of mucin and various mucoadhesive polymers for the measurement of the mucoadhesivity has been reported previously (Suvannasara et al. 2013; Soliman ME et al. 2018). Herein, a simple and reproducible procedure for assessment of mucoadhesiveness of the in situ composite gel was performed through monitoring viscometric changes of the mucin-gel mixture with a Brookfield viscometer (Thirawong et al. 2008; Suvannasara et al. 2013). The viscosity enhancement that was noticed after combining the gel with mucin in the mucin-gel mixture $\left(\eta_{\text {mixture }}\right)$ could be taken as a direct estimate of their interaction occurring in mucoadhesion " $\Delta \mathrm{\eta}$; component of mucoadhesion". 
Our results confirmed such proposed mechanism as revealed from the rheological synergism between mucin and either plain or PV3 loaded gel. The plain and PV3 loaded gels showed component of mucoadhesion of $17382.33 \pm 4810.19$ and $62726.67 \pm 2085.97 \mathrm{cp}$ respectively, suggesting greater synergism and hence, the higher mucoadhesive potential of the in situ composite gel. In addition, PV3 loaded gel displayed a higher ratio of $\Delta \mathrm{\eta} / \eta$ gel (5.18), almost 3.6 fold higher than that for plain gel (1.43). This could be attributed to its content of cationic Eudragit RL100, with the possible formation of electrostatic interaction with anionic gellan gum, allowing high retention of MPs inside the gel matrix.

\section{Morphologic Analysis of the Microstructure of MPs and In Situ Composite Gel}

SEM images of the prepared MPs, shown in Fig. 5 (a-d), confirmed the capability of the selected stabilizers in attaining spherical non-aggregating particles with different surface morphologies. Surface topography of the MPs prepared using P188 and M59 displayed smooth surfaces. Contrastingly, the surfaces of MPs prepared using PVA and T80 were rough. Interestingly, MPs prepared using either PVA or P188 exhibited some small pores.

The microstructure of in situ composite gel is depicted in Fig. 5 (e and f). The plain gel showed irregular and relatively porous microstructure with a smooth surface, whereas PV3 loaded gel was rough indicating the incorporation of MPs in the porous structure of gellan gum gel.

[Figure 5 near here]

\section{In vitro Drug Release and Release Kinetics}

In vitro release profiles of AC from different formulae are illustrated in Fig. 6. AC powder showed complete release in $3 \mathrm{~h}$. AC is a weakly ionizable phenyl acetic acid derivative with pH-dependent solubility, showing an experimentally reported high solubility in phosphate 
buffer pH $6.8\left(10.58 \mathrm{mg} / \mathrm{mL}\right.$, at $37^{\circ} \mathrm{C}$ ) (Usha et al. 2008). Indeed, its weakly acidic nature could guarantee its enhanced dissolution at neutral or slightly alkaline $\mathrm{pH}$ of the simulated salivary fluid.

Expectedly, the release of AC from the control gel was relatively rapid, showing almost $>$ $50 \%$ release in the first hour. However, a somewhat prolongation in the release of AC residual amount was noticed, achieving $100 \%$ release within $8 \mathrm{~h}$. This might be due to the hydration and subsequent pH-dependent swelling behavior of gellan gum (Elmowafy E et al. 2014; Prezotti et al. 2014; Osmałek et al. 2017), facilitating the penetration of dissolution medium and AC diffusion.

In contrast, both selected AC Eudragit RL100 MPs (PV3 MPs) and in situ composite gel (PV3 loaded gel) exhibited a more sustained release manner, reaching $50.61 \%$ and $55.33 \%$ release of AC respectively over $24 \mathrm{~h}$. Slightly higher values of the release of AC were noticed with MPs than its release in in situ composite gel within the first $4 \mathrm{~h}$. The small size of MPs and the presence of pores on their surface, as revealed in SEM image, yielded increased surface area, facilitating the passage of dissolution medium inside the MPs matrix and hence, increasing AC diffusion and release. This indicated that compact gel matrix of in situ composite gel caused more retardation of $\mathrm{AC}$ release in the first $4 \mathrm{~h}$ as compared to MPs alone.

Afterward, a similar non-significant $(\mathrm{p}>0.05)$ trend towards sustained AC fashion was obvious for both formulae. Composite system porosity could guarantee drug leaching from MPs through the pores and subsequent drug release through the gel network (Hoare and Kohane 2008). In addition, the porous network of in situ composite gel could allow the penetration of dissolution medium and might be at the base of lack of variation of AC release from both MPs and MPs 
loaded gel. Moreover, swollen gellan based matrices underwent dissolution, loosening and erosion over time (Elmowafy E et al. 2014), affecting gel strength as stated earlier, exposing AC loaded MPs to the surrounding dissolution medium. Such observed similarity in release profiles of both MPs and their gel systems was previously noticed using different polymers and gel matrices (Karthikeyan et al. 2012; Fedorchak et al. 2017). Such similarity in the release pattern might also provide another evidence of maintaining the integrity of MPs entrapped inside the gel network during gel lyophilization as well as throughout the release period. A matter is of significant benefit for drugs, administered buccally, and of special importance for persistent buccal anti-inflammatory therapy.

The release of AC from Eudragit RL100 MPs and PV3 loaded gel was best fitted by the Higuchi model with the highest linearity $(\mathrm{r} 2=0.957$ and 0.988 respectively) as displayed in Table 4. Fitting to Peppas model, considering the impact of geometry on $\mathrm{n}$ value as welladdressed previously (Bruschi 2015), also exhibited good linearity $(\mathrm{r} 2=0.989$ and 0.992 respectively) with $\mathrm{n}$ values of 0.28 (Quasi-Fickian diffusion for MPs which was tested as a sphere with release exponent of 0.43) and 0.50 (Fickian diffusion for gel which was tested as a thin film with release exponent of 0.5), respectively. Such results indicated diffusion controlled release of AC from both Eudragit RL100 MPs and PV3 loaded gel. This was in agreement with gellan composite matrices (Dewan et al. 2017). On the other hand, the release of AC from control gel followed first order release kinetics as previously observed for gellan gum matrices (Harish et al. 2009; Elmowafy E et al. 2014).

[Figure 6 near here] 


\section{Solid State Characterization}

Differential Scanning Calorimetry (DSC)

DSC thermogram of AC (Fig. 7) depicted its typical melting point $\left(154.6^{\circ} \mathrm{C}\right)$ (Jana, Das, et al. 2013; Patnaik et al. 2015). DSC curve of AC: Eudragit RL100 physical mixture exhibited slight broadening of AC endothermic peak due to the dilution effect. The disappearance of AC peak in PV3 confirmed its molecular dispersion and amorphization within MPs.

The thermogram of gellan gum exhibited an endothermic peak at $64.27{ }^{\circ} \mathrm{C}$ corresponding to the water liberation and an exothermic peak at $245.73^{\circ} \mathrm{C}$ resulting from polymer decomposition. The plain gel was free from characteristic peaks of gellan gum, suggesting gellan gum gelation and cross-linking. Loss of defined thermal events of gellan gum was noticed in the thermograms of plain gel and in situ composite gel, denoting obvious retardation of its degradation in the gel network and higher thermal stability.

[Figure 7 near here]

Fourier Transform Infrared (FT-IR) Spectroscopy

The infrared analysis is usually done to complement the thermal analysis data. It is a very useful technique for evaluating the possible molecular interaction between drugs and particulate matrices such as hydrogen bonding. It was previously postulated that the higher the value of the $\mathrm{OH}$ and $\mathrm{CO}$ stretching peaks shift, the stronger the nature of the intermolecular hydrogen bonding interaction. This could modulate drugs entrapment and release characteristics (Menjoge and Kulkarni 2007; Elmowafy EM et al. 2008; Elmowafy E et al. 2014; Elmowafy E, Gad H, et al. 2019).

The collected spectra of AC, Eudragit RL100, 1:1 physical mixture, selected MPs, gellan gum, plain gel and in situ composite gel are illustrated in Fig. 8. AC spectrum showed 
characteristic peaks of the drug at $3319.48 \mathrm{~cm}^{-1}$ and $3278.5 \mathrm{~cm}^{-1}$ corresponding to secondary N-H vibrations and $\mathrm{O}-\mathrm{H}$ stretching respectively. Peaks at $3033.91 \mathrm{~cm}^{-1}$ and2936.94 $\mathrm{cm}^{-1}$ could be related to aromatic $\mathrm{C}-\mathrm{H}$ stretching vibrations and aliphatic $\mathrm{C}-\mathrm{H}$ stretching vibrations. Sharp peaks at $1771.68 \mathrm{~cm}^{-1}$ and $1452.79 \mathrm{~cm}^{-1}$ were assigned to $\mathrm{C}-\mathrm{O}$ stretching of carboxylic acid and O-H bending respectively. Eudragit RL100 spectrum showed the characteristic band of the carboxylic groups at $1641 \mathrm{~cm}^{-1}$, an absorption band of C-O-C ether linkages at $1106 \mathrm{~cm}^{-1}$, and an absorption band of $\mathrm{NH}_{2}$ at $3487 \mathrm{~cm}^{-1}$. They were in accordance with the current literature (Biswal et al. 2011; Jana, Das, et al. 2013; Fathalla et al. 2014; Jana et al. 2014). Additive spectra of the physical mixture of AC with Eudragit RL100 were confirmed.

In the spectrum of selected PV3 MPs, broadening and shifting of N-H and $\mathrm{OH}$ peaks of AC to $3547.84 \mathrm{~cm}^{-1}$ was revealed, indicating enhanced hydrogen bonding. Shifting of the sharp peak at 1771.68 to a lower intensity peak at $1732.49 \mathrm{~cm}^{-1}$ was another evidence of interaction between AC and Eudragit RL100 at the molecular level, indicating AC entrapment inside MPs. Such interaction between anionic AC and cationic Eudragit RL100 might have an impact on the sustained release of AC from the MPs.

In GG FT-IR spectrum, seyeral characteristic absorbance peaks could be identified. The characteristic $\mathrm{O}-\mathrm{H}$ stretching peak was recorded at $3308.4 \mathrm{~cm}^{-1}$. High-intensity asymmetric COO- stretching and symmetric COO- stretching peaks were revealed at $1607.3 \mathrm{~cm}^{-1}$ and 1414.9 $\mathrm{cm}^{-1}$ respectively. A peak at $1026.71 \mathrm{~cm}^{-1}$ was assigned to C-O stretching.

As for lyophilized plain gel, an obvious shift of C-O stretching peak of gellan gum from 1026.71 $\mathrm{cm}^{-1}$ to $1043.40 \mathrm{~cm}^{-1}$ was reported, indicating gellan gum crosslinking and gelation phenomena. FT-IR is used as an investigative tool to elucidate the interaction between gel and MPs. Freeze dried in situ gel was utilized herein as gel could not be treated as KBr disc (Krokida et al. 2011). 
As revealed in the spectrum of PV3 loaded gel, the O-H stretching peak of gellan gum was shifted to a higher wavenumber from $3308.4 \mathrm{~cm}^{-1}$ to $3449.17 \mathrm{~cm}^{-1}$. As a hydroxyl groupcontaining polymer, gellan gum could form hydrogen bonding with MPs (NH2 of Eudragit polymer) as confirmed by a shift in O-H stretching peak (Elmowafy E et al. 2014). The disappearance of the characteristic band of the carboxylic groups of Eudragit RL100 at $1641 \mathrm{~cm}^{-}$ ${ }^{1}$ could provide evidence support of electrostatic interaction. Interestingly, characteristic peaks of AC at 1771.68 and $1452.79 \mathrm{~cm}^{-1}$ were completely disappeared in MPs loaded gel. This could predict the effective interaction between cationic MPs and anionic gellan gum gel at the molecular level, profoundly sustained AC release. In addition, such interaction might have an implication on the viscosity of the in situ gel which is highly warranted for buccal applicability.

\section{[Figure 8 neâr here]}

\section{In vitro Anti-arthritic Activity}

The albumin anti-denaturation method is used to investigate AC anti-arthritic effect. Nonsteroidal anti-inflammatory drugs have been proved to prevent protein denaturation as well as to block COX enzyme, inhibiting the production of endogenous prostaglandins (Paul 1996).

Both Eudragit RL100 MPs and in situ composite gel possessed good anti-denaturation activity with an obvious dose-dependent response (Fig. 9). Both formulae exhibited IC50 values of 61.14 and $26.2 \mu \mathrm{g} / \mathrm{mL}$ respectively, evidencing effective results comparable to the positive control, diclofenac sodium (IC50 value of $15.12 \mu \mathrm{g} / \mathrm{mL}$ ). It is interesting to note that plain gel showed a minimal response, starting from $125 \mu \mathrm{g} / \mathrm{mL}$. The $\%$ inhibition of protein denaturation for the plain gel was found to be $43.25 \%$ at the highest concentration used $(1000 \mu \mathrm{g} / \mathrm{mL})$, compared to $81.94 \%, 84.63 \%$ and $89.35 \%$ for Eudragit RL100 MPs, in situ composite gel and diclofenac sodium respectively. This might be at the base of higher IC50 value of in situ 
composite gel than that of Eudragit RL100 MPs. Such promising activity of in situ composite gel supports its use as a remedy for arthritis conditions.

\section{[Figure 9 near here]}

\section{In vivo Anti-inflammatory Activity}

Assessment of in vivo anti-inflammatory effect of AC from CMC solution and the in situ composite gel was also done, using plain Na CMC solution and plain in situ gel as control groups. Na CMC aqueous solution was extensively used in literature for in vivo antiinflammatory activity study as a control (Jana, Das, et al. 2013; Jana, Saha, et al. 2013). It was reported to cause no inhibition of inflammatory response produced following the subplantar injection of carrageenan. In this work, motivated by its minimal positive effect on in vitro antiarthritic activity study, the synergistic anti-inflammatory effect of the plain gel was also investigated for comparison purposes. It was observed that in control groups receiving CMC solution and plain gel, the subplantar injection of carrageenan produced local edema, denoting lack of any significant influence on inhibition of inflammatory response $(\mathrm{P}>0.05)$.

Both treated groups, that received standard AC in CMC solution and PV3 loaded gel, displayed prominent inhibitory effect on edema swelling from the first $1 \mathrm{~h}$ and throughout the whole study time as compared to the control group $(\mathrm{p}<0.05)$ (Fig. 10). A slower inhibitory effect was demonstrated in the first $2 \mathrm{~h}$ for in situ composite gel, reaching $35.47 \%$ inhibition of paw edema as compared to AC showing $63.08 \%$ inhibition then became similar in the next $2 \mathrm{~h}$. However, \% inhibition of paw edema was declined after $5 \mathrm{~h}$ for $\mathrm{AC}$ in CMC solution, whereas, in situ composite gel remained superior to $\mathrm{AC}$ over $8 \mathrm{~h}$ after buccal administration. The \% inhibition of PV3 loaded gel was increased by 2.84 fold than AC after $8 \mathrm{~h}(68.07 \%$ versus $23.90 \%$ ), proving its sustained capability in suppressing paw swelling. 
Such an improved and sustained inhibition of inflammatory response may be attributed to the mucoadhesive potential of gellan gum gel reservoir. The proposed mucoadhesiveness of gellan gum was achieved by exerting supramolecular interactions with mucin chains, favoring MPs immobilization and residence in the buccal mucosa. This was profoundly coupled with a continuous local concentration gradient of the entrapped drug over a prolonged period of time (Meneguin et al. 2018; Prezotti et al. 2018).

[Figure 10 near here]

\section{Conclusion}

A buccal delivery system of an in situ composite gellan gum gel containing AC Eudragit RL100 MPs was successfully developed in two steps. The first step aimed to prepare and optimize AC Eudragit RL100 MPs by the emulsion solvent evaporation technique. The second one explored the suitability of in situ ion triggered gellan gum gel to develop promising in situ composite gel. The proposed in situ composite gel not only succeeded in sustaining AC release but also ensured ease of buccal applicability of MPs as well as prolonged localization. A sustained and prolonged drug-effects behavior of MPs loaded gel was demonstrated, relative to standard drug.

\section{Acknowledgments}

The authors would like to acknowledge Evonik Pharma, GmbH, Darmstadt, Germany and E.I.P.I.C.O. Company, Cairo, Egypt for their kind supply of Eudragit RL100 and HPMC respectively. The authors would also like to thank Bristol-Myers Squibb Company, Cairo, Egypt for their kind supply of aceclofenac. 


\section{Funding information}

This manuscript received no sources of financial funding.

\section{Disclosure of interest}

The authors report no conflict of interest

\section{References}

Animesh K, Afrasim M, R Bommareddy R, Ayaz A, Shruthi R, G Shivakumar H. 2012. Applicability and approaches of (Meth) acrylate copolymers (Eudragits) in novel drug delivery systems. Current Drug Therapy. 7(4):219-234.

Biswal I, Dinda A, Mohanty S, Dhara M, Das D, Chowdary K, Si S. 2011. Influence of Drug/Polymer Ratio on the Encapsulation Efficiency of Highly Hydrophilic Drug. Asian Journal of Chemistry. 23(5):1973.

Blanco M, Alonso M. 1997. Development and characterization of protein-loaded poly (lactideco-glycolide) nanospheres. European Journal of Pharmaceutics and Biopharmaceutics. 43(3):287-294.

Boni FI, Prezotti FG, Cury BSF. 2016. Gellan gum microspheres crosslinked with trivalent ion: effect of polymer and crosslinker concentrations on drug release and mucoadhesive properties. Drug development and industrial pharmacy. 42(8):1283-1290.

Bruschi ML. 2015. Strategies to modify the drug release from pharmaceutical systems. Woodhead Publishing.

Chan SY, Goh CF, Lau JY, Tiew YC, Balakrishnan T. 2019. Rice starch thin films as a potential buccal delivery system: Effect of plasticiser and drug loading on drug release profile. International Journal of Pharmaceutics. 562:203-211. 
Cid YP, Pedrazzi V, de Sousa VP, Pierre MBR. 2012. In vitro characterization of chitosan gels for buccal delivery of celecoxib: influence of a penetration enhancer. AAPS PharmSciTech. 13(1):101-111.

Cui F, Qian F, Zhao Z, Yin L, Tang C, Yin C. 2009. Preparation, characterization, and oral delivery of insulin loaded carboxylated chitosan grafted poly (methyl methacrylate) nanoparticles. Biomacromolecules. 10(5):1253-1258.

Dasgupta S, Dey S, Choudhury S, Mazumder B. 2013. Topical delivery of aceclofenac as nanoemulsion comprising excipients having optimum emulsification capabilities: preparation, characterization and in vivo evaluation. Expert opinion on drug delivery. 10(4):411-420.

Dewan M, Sarkar G, Bhowmik M, Das B, Chattoapadhyay AK, Rana D, Chattopadhyay D. 2017. Effect of gellan gum on the thermogelation property and drug release profile of Poloxamer 407 based ophthalmic formulation. International Journal of Biological Macromolecules. 102:258-265.

Dhiman M, Yedurkar P, Sawant KK. 2008. Formulation, characterization, and in vitro evaluation of bioadhesive gels containing 5-fluorouracil. Pharmaceutical development and technology. 13(1):15-25.

El Maghraby GM, Elzayat EM, Alanazi FK. 2012. Development of modified in situ gelling oral liquid sustained release formulation of dextromethorphan. Drug development and industrial pharmacy. 38(8):971-978.

Elisha IL, Dzoyem J-P, McGaw LJ, Botha FS, Eloff JN. 2016. The anti-arthritic, antiinflammatory, antioxidant activity and relationships with total phenolics and total flavonoids of nine South African plants used traditionally to treat arthritis. BMC complementary and alternative medicine. 16(1):307. 
Elkomy MH, El Menshawe SF, Abou-Taleb HA, Elkarmalawy MH. 2017. Loratadine bioavailability via buccal transferosomal gel: formulation, statistical optimization, in vitro/in vivo characterization, and pharmacokinetics in human volunteers. Drug Delivery. 24(1):781-791. Elmowafy E, Abdal-hay A, Skouras A, Tiboni M, Casettari L, Guarino V. 2019. Polyhydroxyalkanoate (PHA): applications in drug delivery and tissue engineering. Expert review of medical devices.(just-accepted).

Elmowafy E, Gad H, Biondo F, Casettari L, Soliman ME. 2019. Exploring optimized methoxy poly (ethylene glycol)-block-poly ( $\varepsilon$-caprolactone) crystalline cored micelles in anti-glaucoma pharmacotherapy. International journal of pharmaceutics.

Elmowafy E, Osman R, AH Ishak R. 2017. Polymer-based novel lung targeted delivery systems. Current pharmaceutical design. 23(3):373-392.

Elmowafy E, Osman R, El-Shamy AE-HA, Awad GA. 2014. Nasal polysaccharides-glucose regulator microparticles: Optimization, tolerability and antidiabetic activity in rats. Carbohydrate polymers. 108:257-265.

Elmowafy E, Soliman ME. 2019. Losartan-chitosan/dextran sulfate microplex as a carrier to lung therapeutics: Dry powder inhalation, aerodynamic profile and pulmonary tolerability. International journal of biological macromolecules.

Elmowafy EM, Awad GA, Mansour S, El-Shamy AE-HA. 2008. Release mechanisms behind polysaccharides-based famotidine controlled release matrix tablets. AAPS PharmSciTech. 9(4):1230-1239.

Elmowafy EM, Tiboni M, Soliman ME. 2019. Biocompatibility, biodegradation and biomedical applications of poly (lactic acid)/poly (lactic-co-glycolic acid) micro and nanoparticles. Journal of Pharmaceutical Investigation.1-34. 
Fathalla D, Abdel-Mageed A, Abdel-Hamid F, Ahmed M. 2014. In-vitro and in-vivo evaluation of niosomal gel containing aceclofenac for sustained drug delivery. International Journal of Pharmaceutical Sciences Research. 2014.

Fedorchak MV, Conner IP, Schuman JS, Cugini A, Little SR. 2017. Long term glaucoma drug delivery using a topically retained gel/microsphere eye drop. Scientific reports. 7(1):8639.

Ferreira IS, Bettencourt A, Bétrisey B, Gonçalves LM, Trampuz A, Almeida AJ. 2015. Improvement of the antibacterial activity of daptomycin-loaded polymeric microparticles by Eudragit RL 100: an assessment by isothermal microcalorimetry. International journal of pharmaceutics. 485(1-2):171-182.

Ferreira IS, Kikhney J, Kursawe L, Kasper S, Gonçalves LM, Trampuz A, Moter A, Bettencourt AF, Almeida AJ. 2018. Encapsulation in Polymeric Microparticles Improves Daptomycin Activity Against Mature Staphylococci Biofilms - a Thermal and Imaging Study. AAPS PharmSciTech. 19(4):1625-1636.

Fini A, Bergamante V, Ceschel GC. 2011. Mucoadhesive gels designed for the controlled release of chlorhexidine in the oral cavity. Pharmaceutics. 3(4):665-679.

Galey WR, Lonsdale H, Nacht S. 1976. The in vitro permeability of skin and buccal mucosa to selected drugs and tritiated water. Journal of Investigative Dermatology. 67(6):713-717.

Garipova V, Gennari C, Selmin F, Cilurzo F, Moustafine R. 2018. Mucoadhesive interpolyelectrolyte complexes for the buccal delivery of clobetasol. Polymers. 10(1):85.

Goodhart FW, Martin AN. 1962. Solubilization of benzoic acid derivatives by polyoxyethylene stearates. Journal of pharmaceutical sciences. 51(1):50-54.

Graciano TB, Coutinho TS, Cressoni CB, de Paula Freitas C, Pierre MBR, de Lima Pereira SA, Shimano MM, da Cunha Frange RC, Garcia MTJ. 2015. Using chitosan gels as a toluidine blue 
O delivery system for photodynamic therapy of buccal cancer: In vitro and in vivo studies. Photodiagnosis and photodynamic therapy. 12(1):98-107.

Harish N, Prabhu P, Charyulu R, Gulzar M, Subrahmanyam E. 2009. Formulation and evaluation of in situ gels containing clotrimazole for oral candidiasis. Indian journal of pharmaceutical sciences. 71(4):421.

Harsh S, Patel K, Padhyay U. 2014. Formulation and evaluation of controlled release colon targeted micro sponge of Aceclofenac. The Pharmacy Innovation Journal. 3(10):81-87.

Hassan EE, Gallo JM. 1990. A simple rheological method for the in vitro assessment of mucinpolymer bioadhesive bond strength. Pharmaceutical research. 7(5):491-495.

Hassan M, Barakat N, El-Badry M, Shehata S. 2011. Formulation and in vitro/in vivo evaluation of naproxen mucoadhesive buccal patches for local effect. Journal of drug delivery science and technology. 21(5):423.

Hoare TR, Kohane DS. 2008. Hydrogels in drug delivery: Progress and challenges. Polymer. 49(8):1993-2007.

Ibrahim E-S, Ismail S, Fetih G, Shaaban O, Hassanein K, Abdellah N. 2012. Development and characterization of thermosensitive pluronic-based metronidazole in situ gelling formulations for vaginal application. Acta pharmaceutica. 62(1):59-70.

Ilić T, Savić S, Batinić B, Marković B, Schmidberger M, Lunter D, Savić M, Savić S. 2018. Combined use of biocompatible nanoemulsions and solid microneedles to improve transport of a model NSAID across the skin: In vitro and in vivo studies. European Journal of Pharmaceutical Sciences. 125:110-119. 
Irimia T, Dinu-Pîrvu C-E, Ghica M, Lupuleasa D, Muntean D-L, Udeanu D, Popa L. 2018. Chitosan-based in situ gels for ocular delivery of therapeutics: A state-of-the-art Review. Marine drugs. 16(10):373.

Jana S, Das A, Nayak AK, Sen KK, Basu SK. 2013. Aceclofenac-loaded unsaturated esterified alginate/gellan gum microspheres: in vitro and in vivo assessment. International journal of biological macromolecules. 57:129-137.

Jana S, Manna S, Nayak AK, Sen KK, Basu SK. 2014. Carbopol gel containing chitosan-egg albumin nanoparticles for transdermal aceclofenac delivery. Colloids and surfaces B: Biointerfaces. 114:36-44.

Jana S, Saha A, Nayak AK, Sen KK, Basu SK. 2013. Aceclofenac-loaded chitosan-tamarind seed polysaccharide interpenetrating polymeric network microparticles. Colloids and Surfaces B: Biointerfaces. 105:303-309.

Karthikeyan K, Durgadevi R, Saravanan K, Shivsankar K, Usha S, Saravanan M. 2012. Formulation of bioadhesive carbomer gel incorporating drug-loaded gelatin microspheres for periodontal therapy. Tropical Journal of Pharmaceutical Research. 11(3):335-343.

Kesavan K, Nath G, Pandit J. 2010. Preparation and in vitro antibacterial evaluation of gatifloxacin mucoadhesive gellan system. Daru: journal of Faculty of Pharmacy, Tehran University of Medical Sciences. 18(4):237.

Khames A. 2019. Hexyl alginate derivative, an amphiphilic innovative buccal film-forming material of promising mechanical and release characteristics for the improvement of repaglinide bioavailability. Drug design, development and therapy. 13:925-940. eng.

Khutoryanskiy VV. 2011. Advances in Mucoadhesion and Mucoadhesive Polymers. Macromolecular Bioscience. 11(6):748-764. 
Krokida M, Pappa A, Agalioti M. 2011. Effect of drying on Aloe's functional components. Procedia Food Science. 1:1523-1527.

Lopez FL, Bowles A, Gul MO, Clapham D, Ernest TB, Tuleu C. 2016. Effect of formulation variables on oral grittiness and preferences of multiparticulate formulations in adult volunteers. European Journal of Pharmaceutical Sciences. 92:156-162.

Madhusudhan S, Panda AK, Parimalakrishnan S, Manavalan R, Manna P. 2010. Design, in vitro and in vivo evaluation of glipizide Eudragit microparticles. Journal of microencapsulation. 27(4):281-291.

Mahdi MH, Conway BR, Mills T, Smith AM. 2016. Gellan gum fluid gels for topical administration of diclofenac. International journal of pharmaceutics. 515(1):535-542.

Mahdi MH, Conway BR, Smith AM. 2014. Evaluation of gellan gum fluid gels as modified release oral liquids. International journal of pharmaceutics.475(1):335-343.

Meneguin AB, Beyssac E, Garrait G, Hsein H, Cury BS. 2018. Retrograded starch/pectin coated gellan gum-microparticles for oral administration of insulin: A technological platform for protection against enzymatic degradation and improvement of intestinal permeability. European Journal of Pharmaceutics and Biopharmaceutics. 123:84-94.

Menjoge A, Kulkarni-M. 2007. Mechanistic investigations of phase behavior in Eudragit ${ }^{\circ}$ E blends. International journal of pharmaceutics. 343(1-2):106-121.

Miyazaki S, Aoyama H, Kawasaki N, Kubo W, Attwood D. 1999. In situ-gelling gellan formulations as vehicles for oral drug delivery. Journal of Controlled Release. 60(2):287-295.

Momoh M, Kenechukwu F, Adedokun M, Odo C, Attama A. 2014. Pharmacodynamics of diclofenac from novel Eudragit entrapped microspheres. Drug delivery. 21(3):193-203. 
Morales JO, Su R, McConville JT. 2013. The influence of recrystallized caffeine on waterswellable polymethacrylate mucoadhesive buccal films. AAPS PharmSciTech. 14(2):475-484.

Mouftah S, Abdel-Mottaleb MM, Lamprecht A. 2016. Buccal delivery of low molecular weight heparin by cationic polymethacrylate nanoparticles. International journal of pharmaceutics. 515(1-2):565-574.

Murakami H, Kawashima Y, Niwa T, Hino T, Takeuchi H, Kobayashi M. 1997. Influence of the degrees of hydrolyzation and polymerization of poly (vinylalcohol) on the preparation and properties of poly (DL-lactide-co-glycolide) nanoparticle. International journal of pharmaceutics. 149(1):43-49.

Nagarwal RC, Pandit J. 2008. Phase transition system: novel oral in-situ gel. Current drug delivery. 5(4):282-289.

Nazari K, Kontogiannidou E, Ahmad RH, Gratsani A, Rasekh M, Arshad MS, Sunar BS, Armitage D, Bouropoulos N, Chang M-W. 2017. Development and characterisation of cellulose based electrospun mats for buccal delivery of non-steroidal anti-inflammatory drug (NSAID). European Journal of Pharmaceutical Sciences. 102:147-155.

Osmałek T, Milanowski B, Froelich A, Szybowicz M, Białowąs W, Kapela M, Gadziński P, Ancukiewicz K. 2017. Design and characteristics of gellan gum beads for modified release of meloxicam. Drug Development and Industrial Pharmacy. 43(8):1314-1329.

Patel B, Banwait H, Parmar K, Patel M. 2011. Formulation and evaluation of topical aceclofenac gel using different gelling agent. International Journal of drug development and research.

Patel V, Prajapat B, Patel J, Patel M. 2006. Physicochemical characterization and evaluation of buccal adhesive patches containing propranolol hydrochloride. Current drug delivery. 3(3):325331. 
Patel VF, Liu F, Brown MB. 2011. Advances in oral transmucosal drug delivery. Journal of controlled release. 153(2):106-116.

Patil HG, Tiwari RV, Repka MA, Singh KK. 2016. Formulation and development of orodispersible sustained release tablet of domperidone. Drug development and industrial pharmacy. 42(6):906-915.

Patnaik S, Aditha SK, Rattan T, Kamisetti V. 2015. Aceclofenac-Soluplus® Nanocomposites for Increased Bioavailability. Soft Nanoscience Letters. 5(02):13.

Paul A. 1996. Analgesic-antipyretics and antiinflammatory agents and drugs employed in the treatment of gout. In The Pharmacological Basis of Therapeutics.617-657.

Perioli L, Ambrogi V, Angelici F, Ricci M, Giovagnoli S, Capuccella M, Rossi C. 2004. Development of mucoadhesive patches for buccal administration of ibuprofen. Journal of controlled release. 99(1):73-82.

Perioli L, Ambrogi V, Giovagnoli S, Ricci M, Blasi P, Rossi C. 2007. Mucoadhesive bilayered tablets for buccal sustained release of flurbiprofen. AAPS PharmSciTech. 8(3):E20-E27.

Prezotti FG, Boni FI, Ferreira NN, Campana-Filho SP, Almeida A, Vasconcelos T, Gremião MPD, Cury BSF, Sarmento B. 2018. Gellan Gum/Pectin Beads Are Safe and Efficient for the Targeted Colonic Delivery of Resveratrol. Polymers. 10(1):50.

Prezotti FG, Cury BSF, Evangelista RC. 2014. Mucoadhesive beads of gellan gum/pectin intended to controlled delivery of drugs. Carbohydrate polymers. 113:286-295.

Raj R, Mongia P, Ram A, Jain N. 2016. Enhanced skin delivery of aceclofenac via hydrogelbased solid lipid nanoparticles. Artificial cells, nanomedicine, and biotechnology. 44(6):14341439. 
Reddy PC, Chaitanya K, Rao YM. 2011. A review on bioadhesive buccal drug delivery systems: current status of formulation and evaluation methods. DARU Journal of Pharmaceutical Sciences. 19(6):385.

Russo E, Selmin F, Baldassari S, Gennari CGM, Caviglioli G, Cilurzo F, Minghetti P, Parodi B. 2016. A focus on mucoadhesive polymers and their application in buccal dosage forms. Journal of Drug Delivery Science and Technology. 32:113-125.

Sanghai P, Nandgude T, Poddar S. 2016. Formulation of bilayer benzydamine $\mathrm{HCl}$ patch targeted for gingivitis. Journal of drug delivery. 2016.

Sharma N, Madan P, Lin S. 2016. Effect of process and formulation variables on the preparation of parenteral paclitaxel-loaded biodegradable polymeric nanoparticles: A co-surfactant study. asian journal of pharmaceutical sciences. 11(3):404-416.

Soliman ME, Elmowafy E, Casettari L, Alexander C. 2018. Star-shaped poly (oligoethylene glycol) copolymer-based gels: Thermo-responsive behaviour and bioapplicability for risedronate intranasal delivery. International journal of pharmaceutics. 543(1-2):224-233.

Soliman ME, Elmowafy, E.M., Almogerbi, A., Mansour, S.A., \& Shamy, A.A. 2016. Dual buoyant / mucoadhesive macroporous polypropylene microparticles for gastric delivery of Repaglinide. international journal of drug delivery. 7(4):197-207.

Srinivas S, Kumar YA, Hemanth A, Anitha M. 2010. Preparation and evaluation of niosomes containing aceclofenac. Dig J Nanomater Bios. 5(1):249-254.

Sudhakar Y, Kuotsu K, Bandyopadhyay A. 2006. Buccal bioadhesive drug delivery-a promising option for orally less efficient drugs. Journal of controlled release. 114(1):15-40. 
Suresh PK, Manhar S. 2014. Bioadhesive buccal gels impregnated with fluconazole: formulation, in vitro and ex vivo characterization. Journal of Applied Pharmaceutical Science. 4(3):15.

Suvannasara P, Juntapram K, Praphairaksit N, Siralertmukul K, Muangsin N. 2013. Mucoadhesive 4-carboxybenzenesulfonamide-chitosan with antibacterial properties. Carbohydrate polymers. 94(1):244-252.

Swain GP, Patel S, Gandhi J, Shah P. 2019. Development of Moxifloxacin Hydrochloride loaded in-situ gel for the treatment of periodontitis: In-vitro drug release study and antibacterial activity. Journal of oral biology and craniofacial research. 9(3):190-200.

Thirawong N, Kennedy RA, Sriamornsak P. 2008. Viscometric study of pectin-mucin interaction and its mucoadhesive bond strength. Carbohydrate Polymers. 71(2):170-179.

Usha AN, Mutalik S, Reddy MS, Ranjith AK, Kushtagi P, Udupa N. 2008. Preparation and, in vitro, preclinical and clinical studies of aceclofenac spherical agglomerates. European journal of pharmaceutics and biopharmaceutics. 70(2):674-683.

Wan LS, Lee PF. 1974. CMC of polysorbates. Journal of Pharmaceutical Sciences. 63(1):136137.

Xie H, Li L, Sun Y, Wang Y, Gao S, Tian Y, Ma X, Guo C, Bo F, Zhang L. 2019. An Available Strategy for Nasal Brain Transport of Nanocomposite Based on PAMAM Dendrimers via In Situ Gel. Nanomaterials. 9(2):147.

Zhu L, Ao J, Li P. 2015. A novel in situ gel base of deacetylase gellan gum for sustained ophthalmic drug delivery of ketotifen: in vitro and in vivo evaluation. Drug design, development and therapy. 9:3943. 


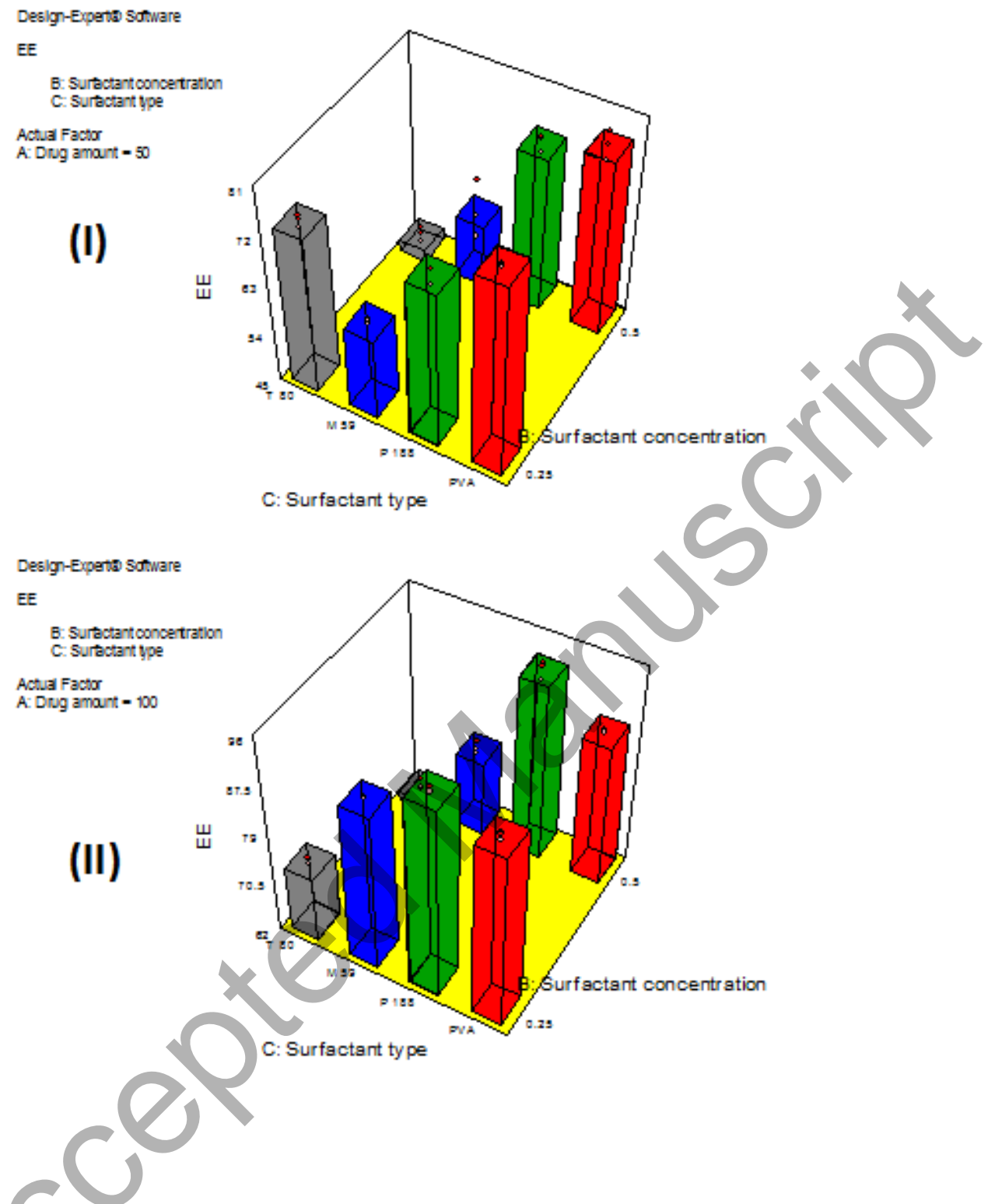

Fig.1. 3D surface plots of EE\% response for drug amounts (I) $50 \mathrm{mg}$ and (II) $100 \mathrm{mg}$ 

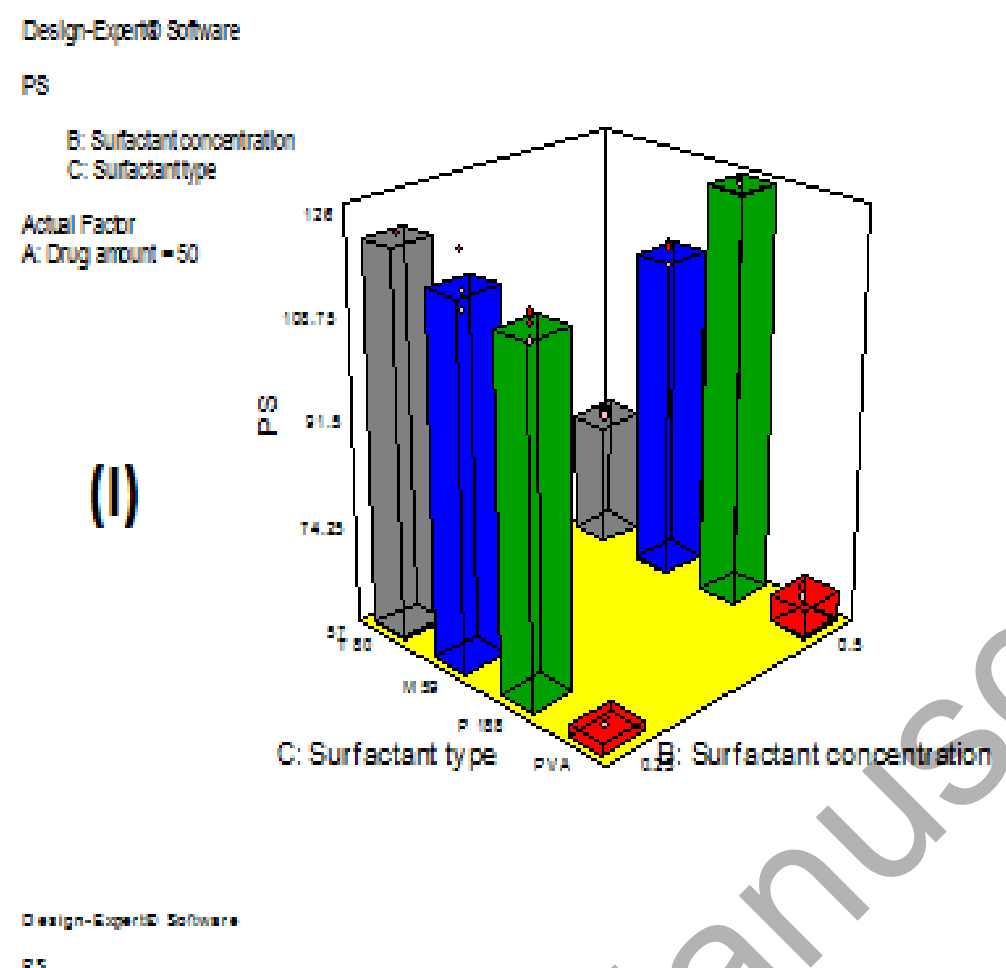

ps

Q: Suracianteancentration

C: Suriciantivo.

Actal Facter

A: Orug amaunt * 100
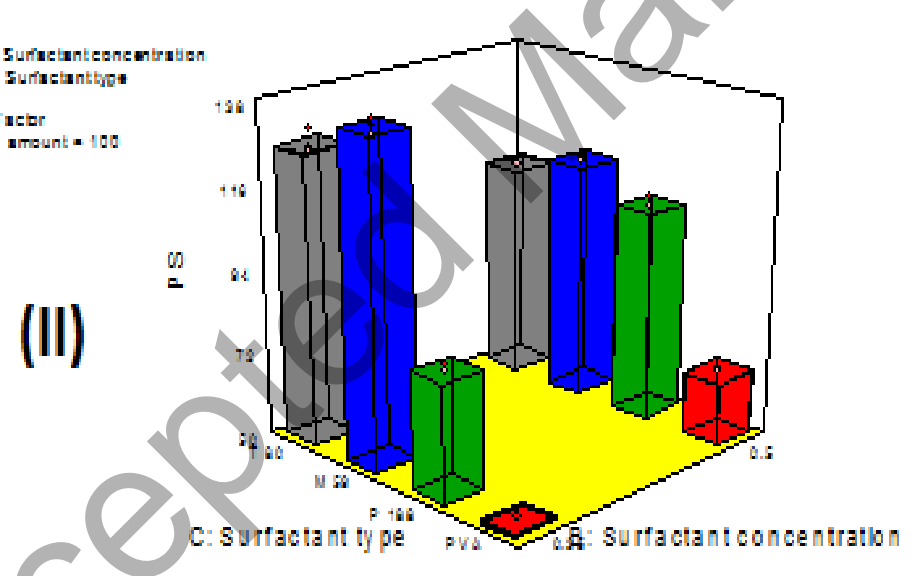

Fig.2. 3D surface plots of PS response for drug amounts (I) $50 \mathrm{mg}$ and (II) $100 \mathrm{mg}$ 

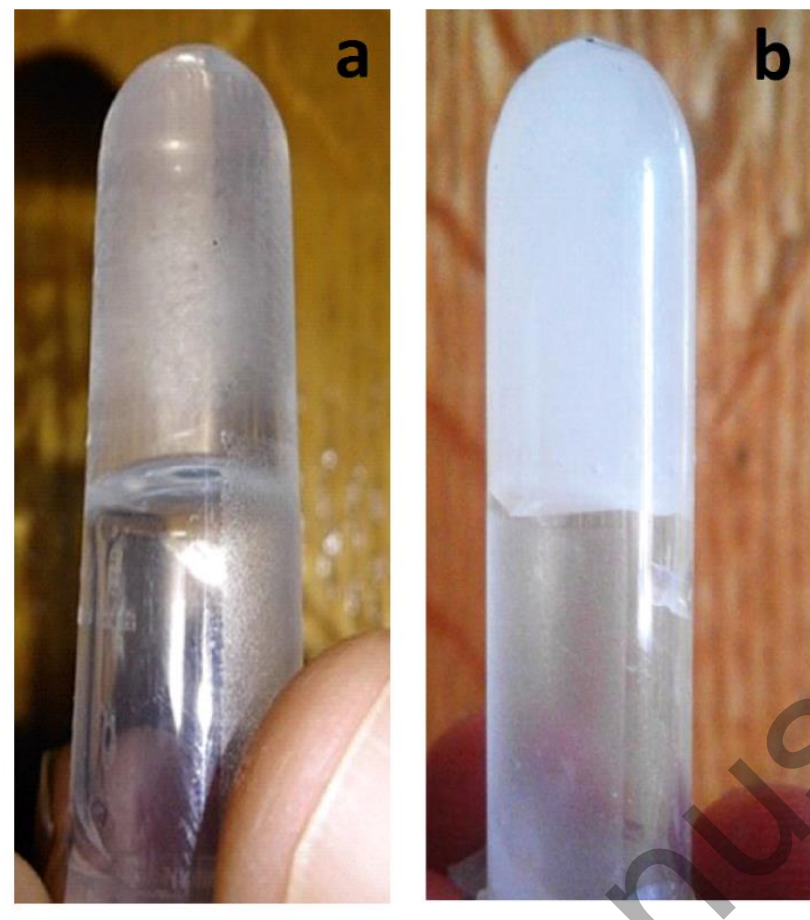

Fig.3. Sol-gel phase transition of in situ plain (a) and composite (b) gel at $37^{\circ} \mathrm{C}$ upon contact with simulated salivary fluid pH 6.8

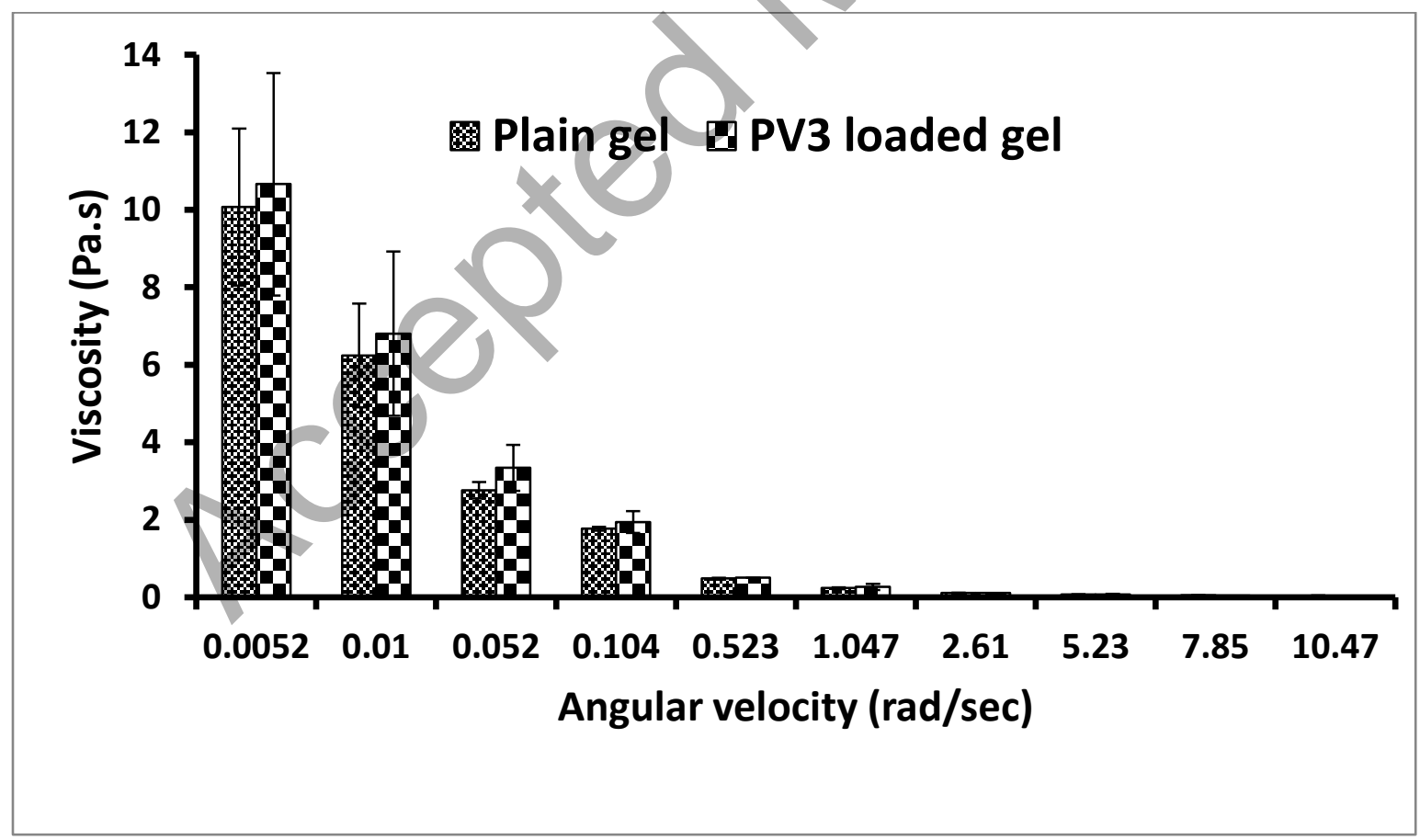

Fig. 4. Viscosity measurements of the investigated plain gel and PV3 loaded gel. Experiments were performed in triplicate; data are presented as average values \pm standard deviation. 

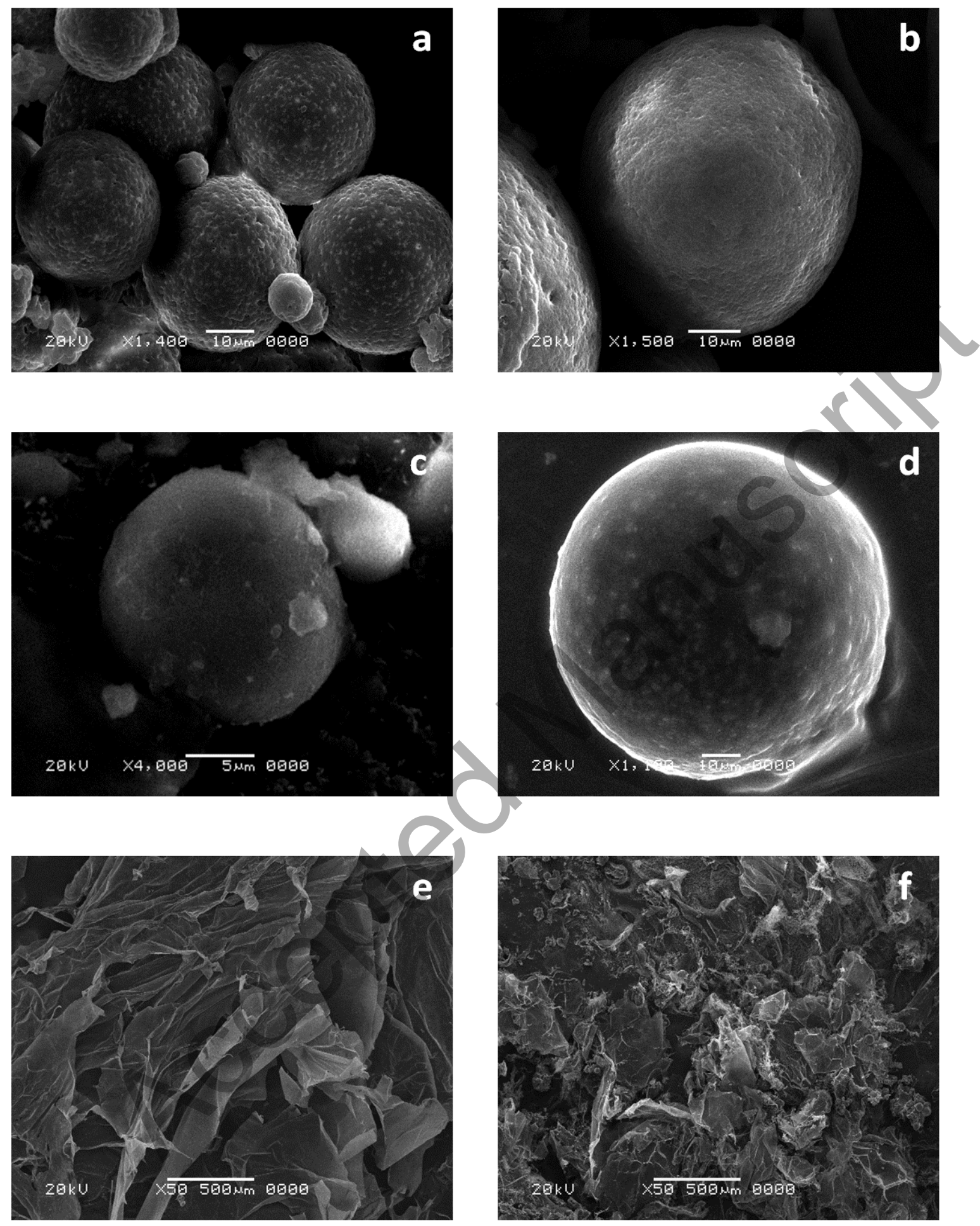

Fig. 5. SEM of representative aceclofenac microparticles using different stabilizers (a) PVA, (b) P 188, (c) M 59 and (d) T 80, and in situ composite gel (e) plain gel and (f) PV3 loaded gel (magnification power X 1400, $1500,4000,1100,50$ and 50 for a-f respectively) 


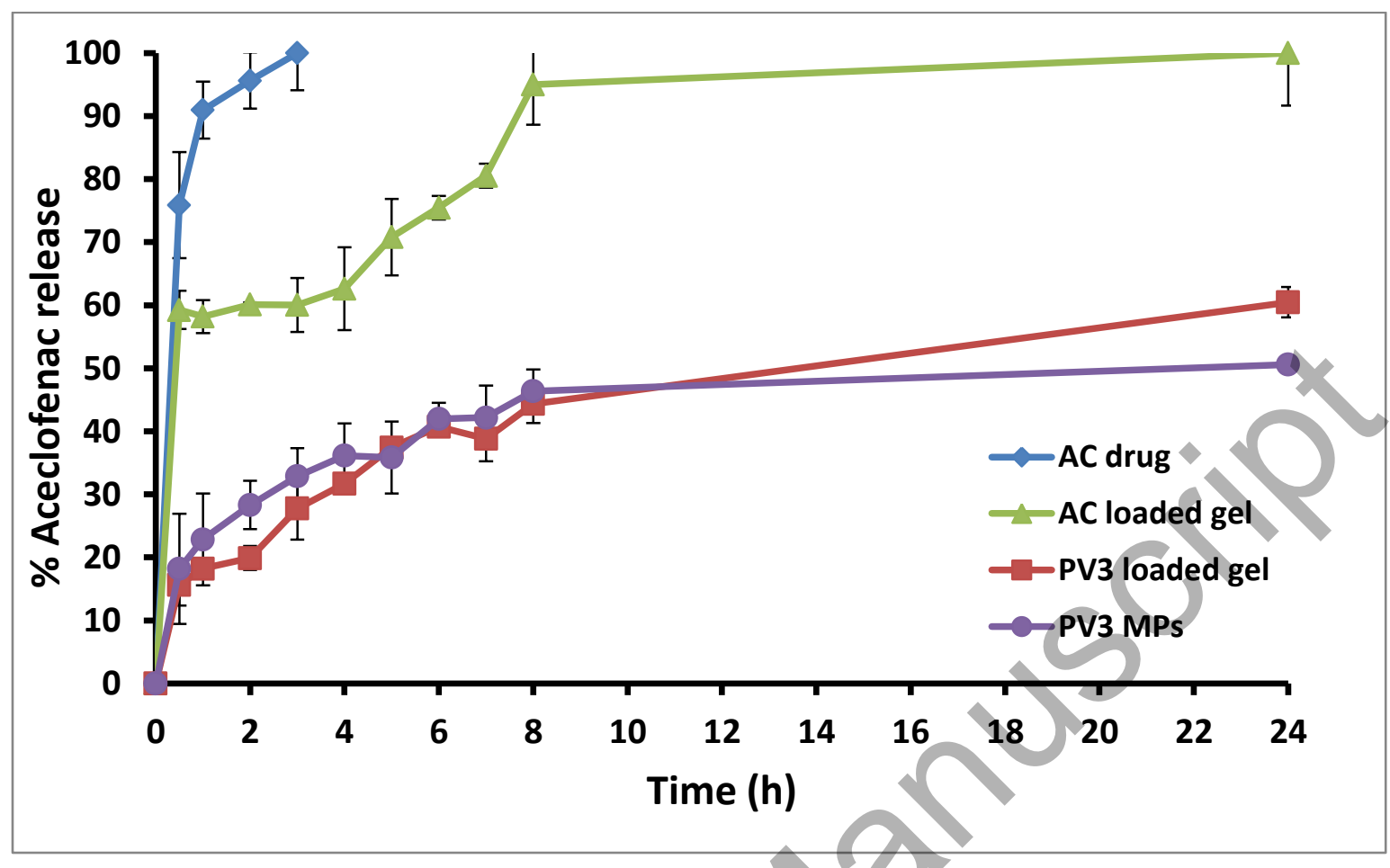

Fig. 6. In vitro release pattern of different aceclofenac formulations. Experiments were performed in triplicate; data are presented as average values \pm standard deviation. 


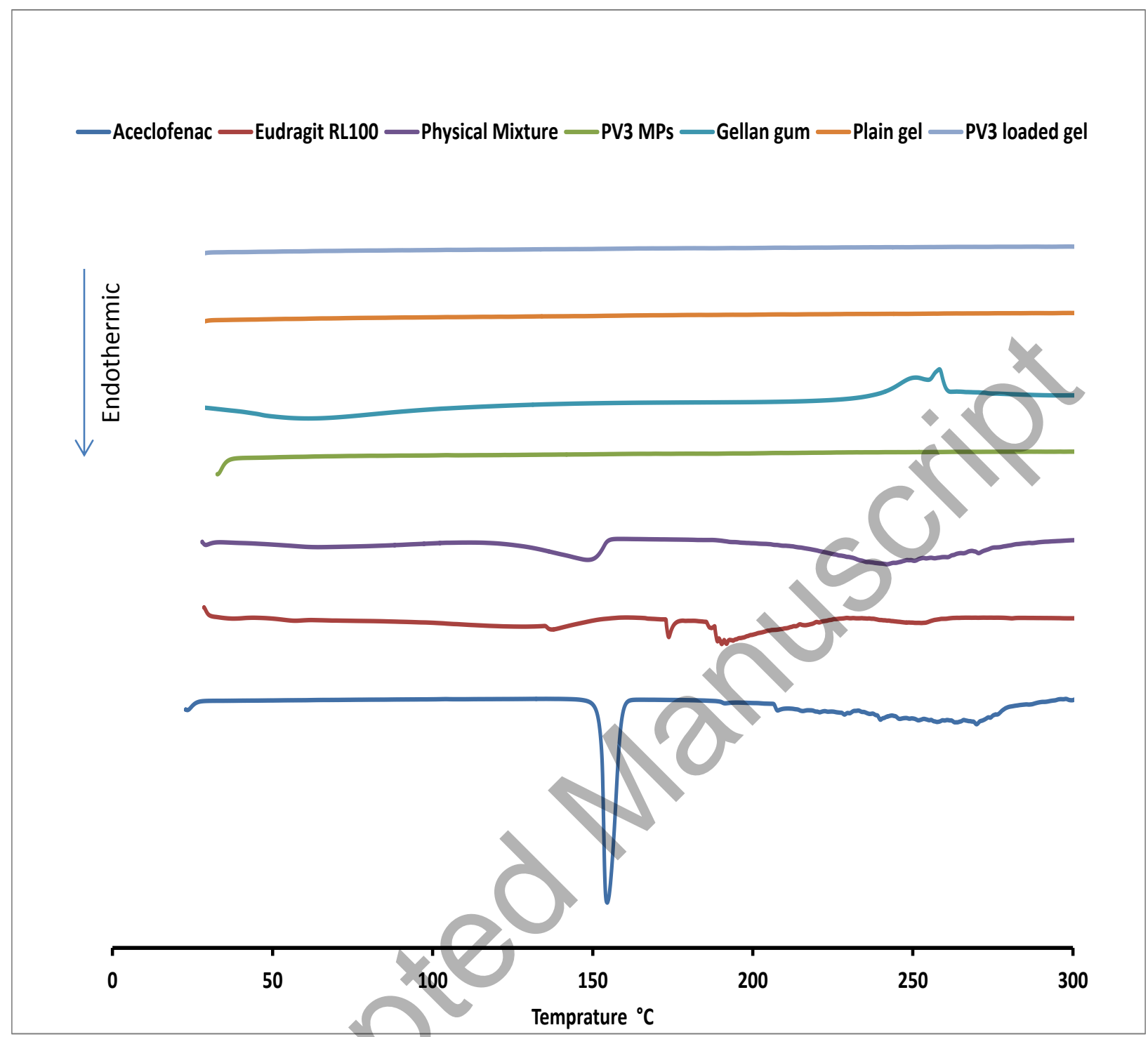

Fig. 7. DSC thermograms of aceclofenac, eudragit RL100, 1:1 physical mixture, PV3 MPs, gellan gum, plain gel and PV3 loaded gel 


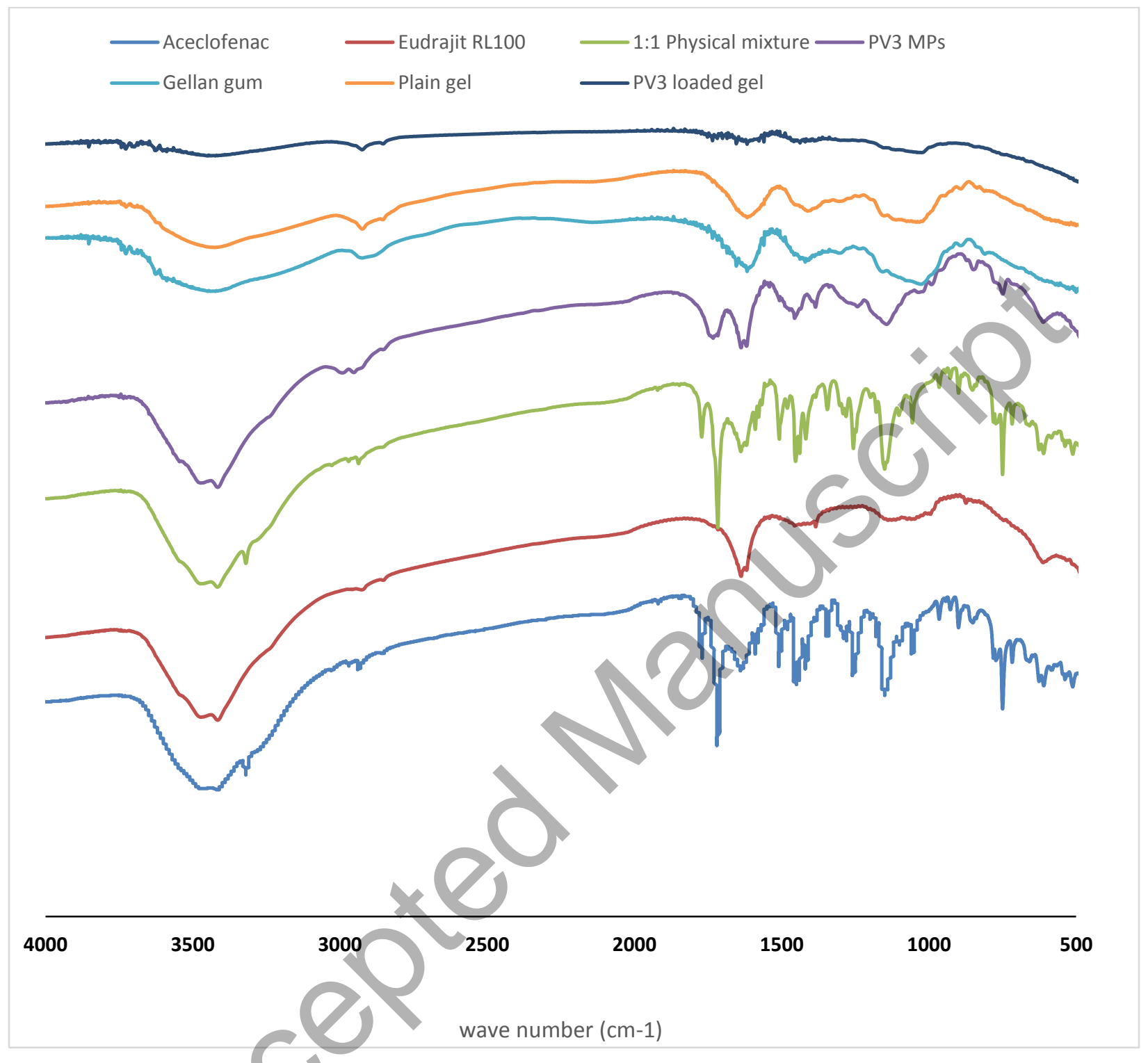

Fig. 8. FT-IR spectra of aceclofenac, eudragit RL100, 1:1 physical mixture, PV3 MPs, gellan gum, plain gel and PV3 loaded gel 


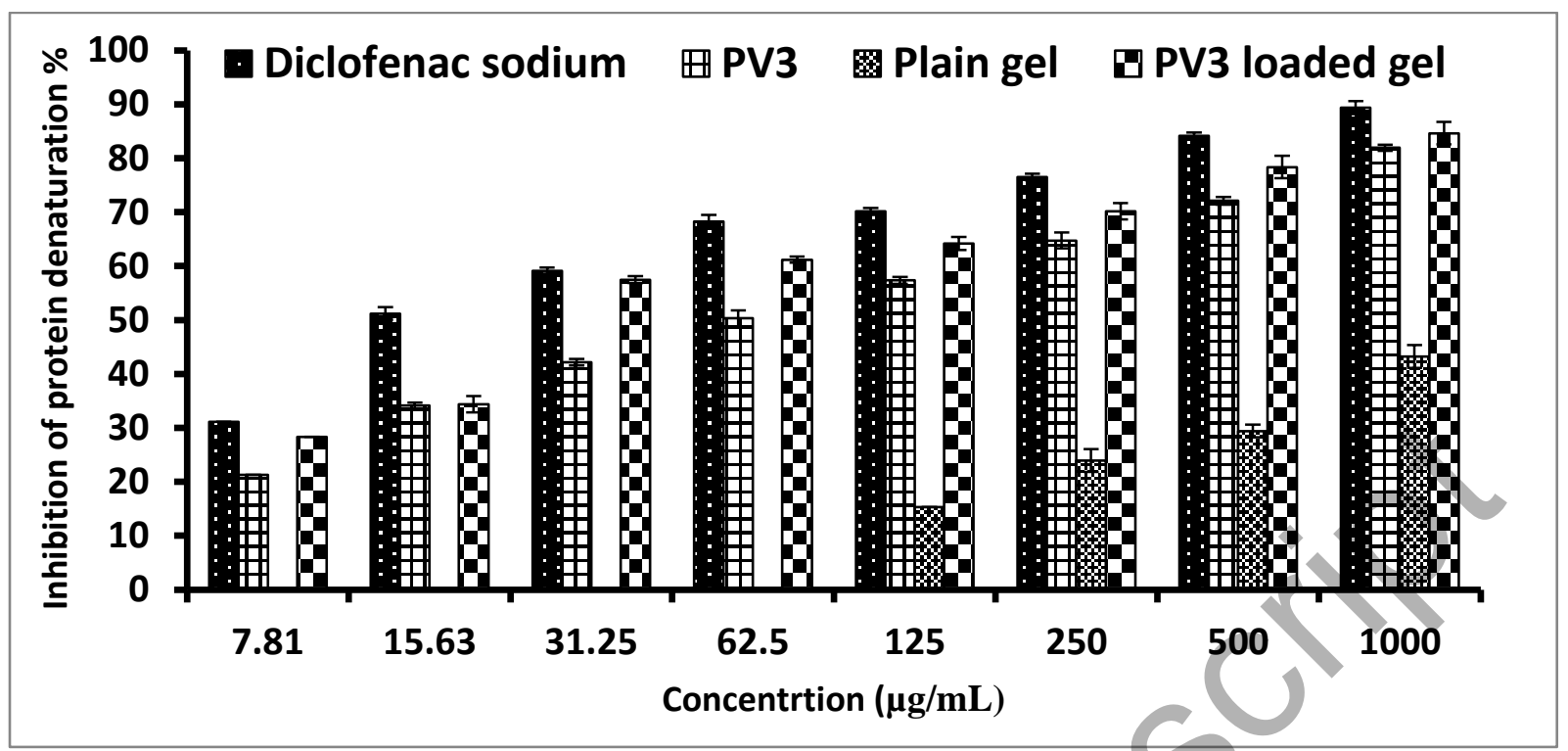

Fig. 9. Anti-arthritic activity of PV3 loaded gel (compared to PV3 MPs and plain gel) and diclofenac sodium as positive control. Experiments were performed in triplicate; data are presented as average values \pm standard deviation. Concentration values were for diclofenac and aceclofenac drugs. The \% inhibition for plain gel was tested for gel formulation without adding PV3 MPs.

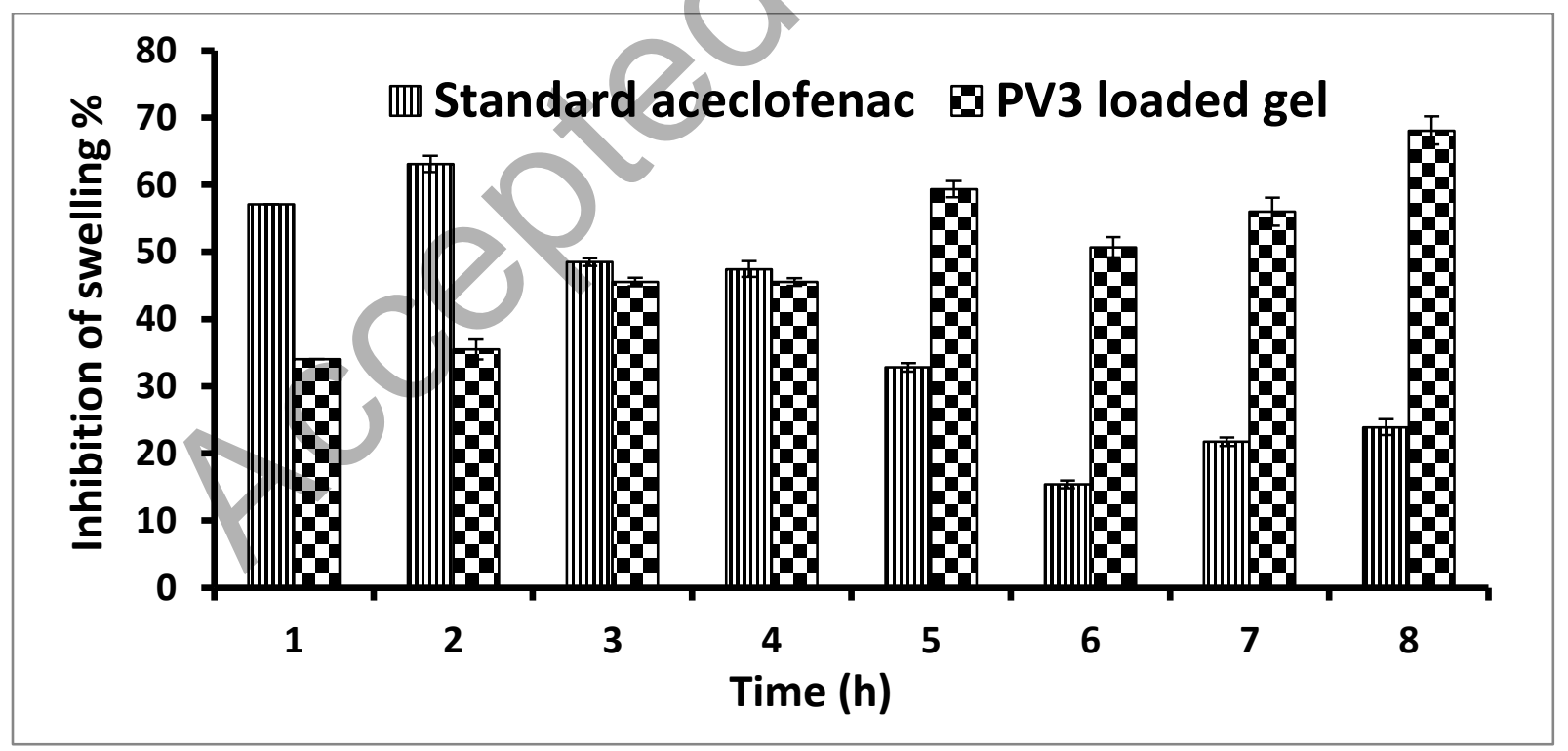

Fig. 10. The percentage inhibition of paw edema swelling of PV3 loaded gel (test) compared to aceclofenac (standard). Experiments were performed on 6 rats; data are presented as average values \pm standard deviation. 


\section{Figure Captions}

Fig.1. 3D surface plots of EE\% response for drug amounts (I) $50 \mathrm{mg}$ and (II) $100 \mathrm{mg}$

Fig.2. 3D surface plots of PS response for drug amounts (I) $50 \mathrm{mg}$ and (II) $100 \mathrm{mg}$

Fig.3. Fig.3. Sol-gel phase transition of in situ plain (a) and composite (b) gel at $37{ }^{\circ} \mathrm{C}$ upon contact with simulated salivary fluid pH 6.8

Fig. 4. Viscosity measurements of the investigated plain gel and PV3 loaded gel. Experiments were performed in triplicate; data are presented as average values \pm standard deviation.

Fig. 5. SEM of representative aceclofenac microparticles using different stabilizers (a) PVA, (b) P 188, (c) M 59 and (d) T 80, and in situ composite gel (e) plain gel and (f) PV3 loaded gel

Fig. 6. In vitro release pattern of different aceclofenac formulations. Experiments were performed in triplicate; data are presented as average values \pm standard deviation.

Fig. 7. DSC thermograms of aceclofenac, eudragit RL100, 1:1 physical mixture, PV3 MPs, gellan gum, plain gel and PV3 loaded gel

Fig. 8. FT-IR spectra of aceclofenac, eudragit RL100, 1:1 physical mixture, PV3 MPs, gellan gum, plain gel and PV3 loaded gel

Fig. 9. Anti-arthritic activity of PV3 loaded gel (compared to PV3 MPs and plain gel) and diclofenac sodium as positive control. Experiments were performed in triplicate; data are presented as average values \pm standard deviation. Concentration values were for diclofenac and aceclofenac drugs. The \% inhibition for plain gel was tested for gel formulation without adding PV3 MPs.

Fig. 10. The percentage inhibition of paw edema swelling of PV3 loaded gel (test) compared to aceclofenac (standard). Experiments were performed on 6 rats; data are presented as average values \pm standard deviation. 
Table 1: $\mathbf{2}^{\mathbf{2}} \mathbf{4}^{\mathbf{1}}$ full factorial design

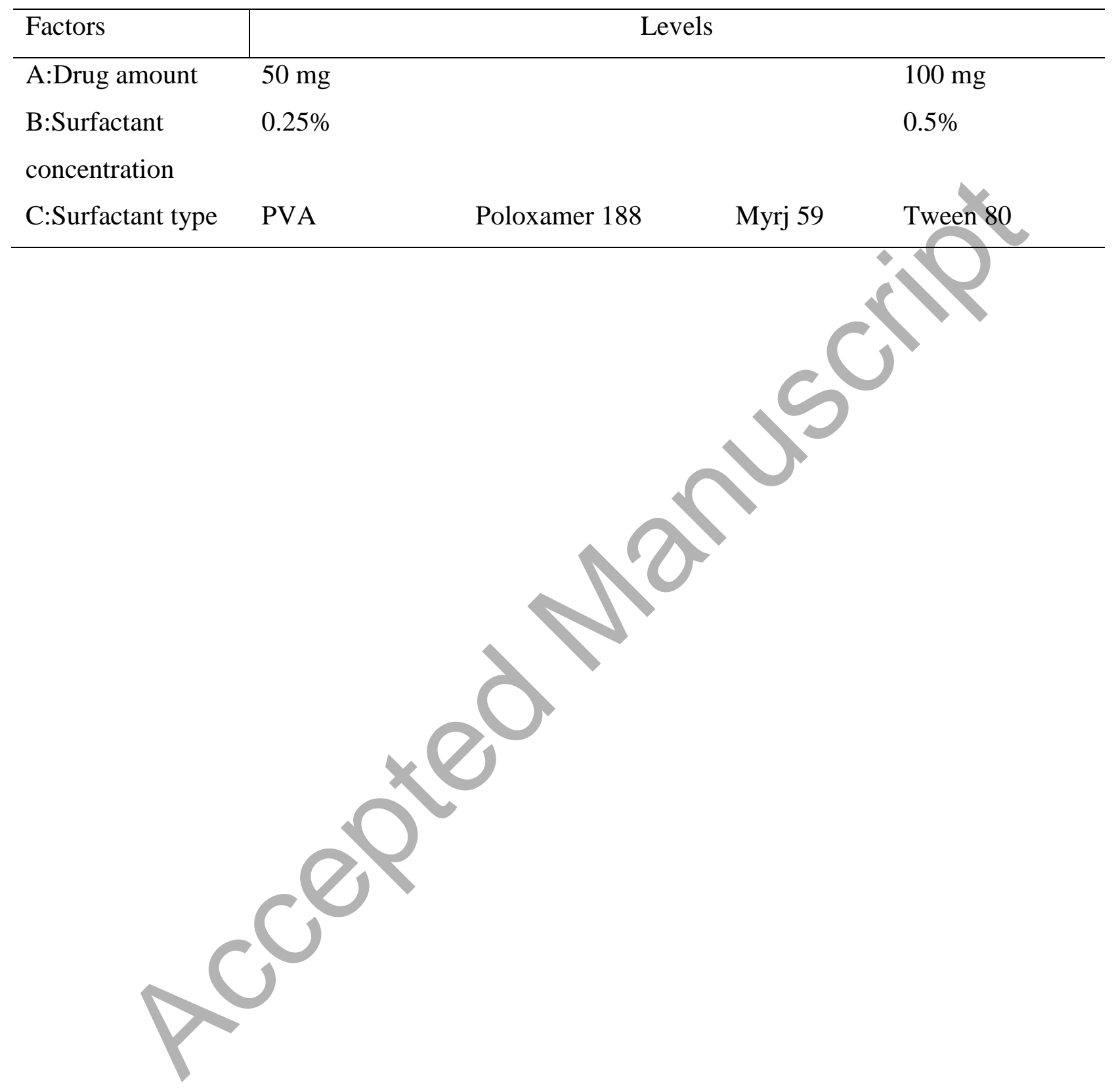


Table 2: composition of prepared aceclofenac microparticles according to the the $2^{2} 4^{1}$ full factorial design and their measured responses

\begin{tabular}{llllll}
\hline MPs & \multicolumn{3}{c}{ Independent variables } & \multicolumn{2}{c}{ Responses } \\
code & A:Drug & B:Surfactant & C:Surfactant & Y $\mathbf{1}$ EE\% & $\mathbf{Y}_{2}:$ PS $(\boldsymbol{\mu m})$ \\
& amount & concentration & type & & \\
& $(\mathbf{m g})$ & $\mathbf{( \% )}$ & & & \\
\hline PV1 & 50 & 0.25 & PVA & $80.44 \pm 0.32$ & $58.75 \pm 0.71$ \\
P1 & 50 & 0.25 & Poloxamer 188 & $74.25 \pm 1.78$ & $114.53 \pm 2.14$ \\
M1 & 50 & 0.25 & Myrj 59 & $60.39 \pm 0.57$ & $116.83 \pm 4.72$ \\
T1 & 50 & 0.25 & Tween 80 & $73.75 \pm 1.02$ & $120.72 \pm 0.37$ \\
PV2 & 50 & 0.5 & PVA & $77.08 \pm 2.52$ & $61.75 \pm 0.87$ \\
P2 & 50 & 0.5 & Poloxamer 188 & $73.42 \pm 1.58$ & $125.44 \pm 0.40$ \\
M2 & 50 & 0.5 & Myrj 59 & $56.42 \pm 5.52$ & $110.38 \pm 2.08$ \\
T2 & 50 & 0.5 & Tween 80 & $47.14 \pm 1.47$ & $77.90 \pm 0.70$ \\
PV3 & 100 & 0.25 & PVA & $94.73 \pm 0.43$ & $51.00 \pm 0.87$ \\
P3 & 100 & 0.25 & Poloxamer 188 & $92.09 \pm 0.66$ & $80.74 \pm 1.31$ \\
M3 & 100 & 0.25 & Myrj 59 & $88.96 \pm 0.22$ & $135.39 \pm 2.02$ \\
T3 & 100 & 0.25 & Tween 80 & $73.37 \pm 0.64$ & $126.52 \pm 3.04$ \\
PV4 & 100 & 0.5 & PVA & $86.32 \pm 0.27$ & $66.78 \pm 0.16$ \\
P4 & 100 & 0.5 & Poloxamer 188 & $92.34 \pm 1.39$ & $105.65 \pm 1.69$ \\
M4 & 100 & 0.5 & Myrj 59 & $89.60 \pm 0.42$ & $112.92 \pm 1.13$ \\
T4 & 100 & 0.5 & Tween 80 & $63.57 \pm 0.97$ & $106.72 \pm 0.73$ \\
\hline
\end{tabular}

(Experiments were performed in triplicate; data are presented as average values \pm standard deviation "SD") 
Table 3: Sum of squares and F-value for the measured responses

\begin{tabular}{lllll}
\hline Model term & \multicolumn{2}{c}{ Sum of squares } & \multicolumn{2}{c}{ F-value } \\
& EE\% & PS & EE\% & PS \\
\hline Model & 8421.35 & 34021.50 & 176.48 & 699.91 \\
A & 2871.21 & $8.164^{*} 10^{-3}$ & $901.28^{\mathrm{s}}$ & $2.519^{*} 10^{-3}$ \\
B & 829.84 & 262.15 & $260.85^{\mathrm{s}}$ & $80.89^{\mathrm{s}}$ \\
C & 3461.33 & 25041.50 & $362.68^{\mathrm{s}}$ & $2575.83^{\mathrm{s}}$ \\
AB & 1.37 & 208.87 & 0.43 & $64.45^{\mathrm{s}}$ \\
AC & 495.06 & 3358.33 & $51.87^{\mathrm{s}}$ & $345.45^{\mathrm{s}}$ \\
BC & 474.81 & 4508.09 & $49.75^{\mathrm{s}}$ & $463.71^{\mathrm{s}}$ \\
ABC & 291.73 & 642.56 & $30.57^{\mathrm{s}}$ & $214.19^{\mathrm{s}}$ \\
\hline
\end{tabular}

${ }^{\mathrm{s}}$ significant at $\mathrm{P}<0.001$

Table 4: Release kinetics of selected MPs and in situ composite gel

\begin{tabular}{|c|c|c|c|}
\hline \multirow[t]{2}{*}{ Formula } & First order $\quad$ Zero order & Higuchi & Peppas \\
\hline & $\mathbf{R}^{2}$ & $\mathbf{R}^{2}$ & $\mathbf{R}^{2}$ (n value) \\
\hline PV3 MPs & 20.936 & 0.957 & $0.989(\mathrm{n}=0.28)$ \\
\hline $\begin{array}{l}\text { PV3 loaded in } \\
\text { situ gel }\end{array}$ & 0.843 & 0.988 & $0.992(n=0.5)$ \\
\hline
\end{tabular}




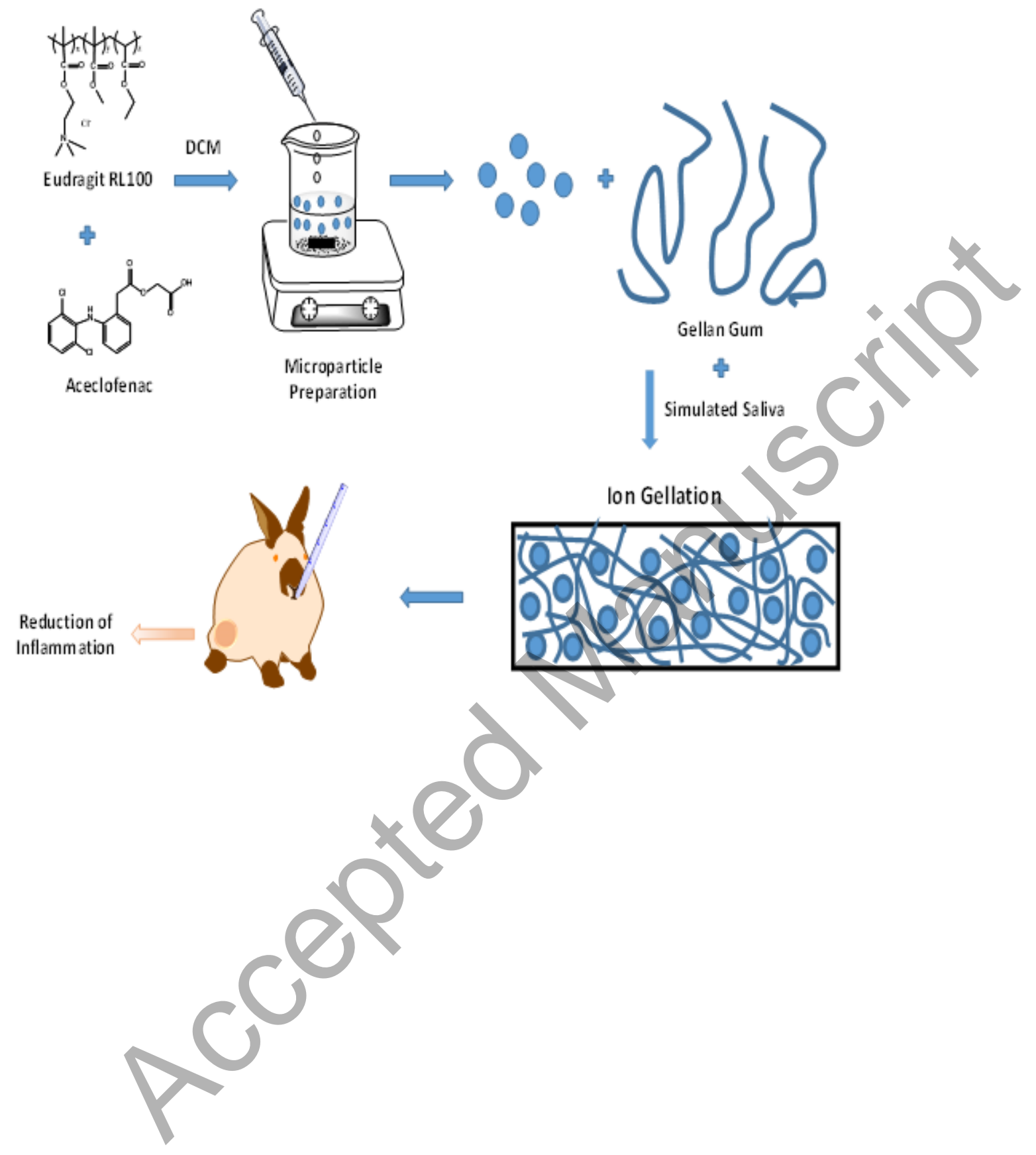

